\title{
The R-Ras/RIN2/Rab5 complex controls endothelial cell adhesion and morphogenesis via active integrin endocytosis and Rac signaling
}

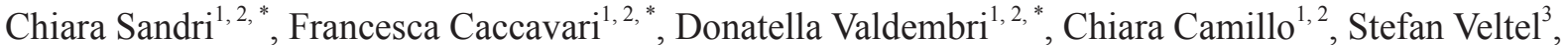 \\ Martina Santambrogio ${ }^{1,2}$, Letizia Lanzetti ${ }^{2,4}$, Federico Bussolino ${ }^{2,5,6}$, Johanna Ivaska ${ }^{3}$, Guido Serini ${ }^{1,2,6}$ \\ ${ }^{1}$ Laboratory of Cell Adhesion Dynamics, Institute for Cancer Research and Treatment (IRCC), Strada Provinciale 142, Km 3.95, \\ 10060 Candiolo (TO), Italy; ${ }^{2}$ Department of Oncological Sciences, University of Torino School of Medicine, 10060 Candiolo (TO), \\ Italy; ${ }^{3}$ University of Turku Centre for Biotechnology and VTT Medical Biotechnology, FIN-20520, Turku, Finland; ${ }^{4}$ Laboratory of \\ Membrane Trafficking, Institute for Cancer Research and Treatment (IRCC), 10060 Candiolo (TO), Italy; ${ }^{5}$ Laboratory of Vascular \\ Oncology, Institute for Cancer Research and Treatment (IRCC), 10060 Candiolo (TO), Italy; ${ }^{6}$ Center for Complex Systems in Mo- \\ lecular Biology and Medicine - SysBioM - University of Torino, clo Department of Animal and Human Biology (DBAU), 10123 \\ Torino, Italy
}

During developmental and tumor angiogenesis, semaphorins regulate blood vessel navigation by signaling through plexin receptors that inhibit the R-Ras subfamily of small GTPases. R-Ras is mainly expressed in vascular cells, where it induces adhesion to the extracellular matrix (ECM) through unknown mechanisms. We identify the Ras and Rab5 interacting protein RIN2 as a key effector that in endothelial cells interacts with and mediates the pro-adhesive and -angiogenic activity of R-Ras. Both R-Ras-GTP and RIN2 localize at nascent ECM adhesion sites associated with lamellipodia. Upon binding, GTP-loaded R-Ras converts RIN2 from a Rab5 guanine nucleotide exchange factor (GEF) to an adaptor that first interacts at high affinity with Rab5-GTP to promote the selective endocytosis of ligand-bound/ active $\beta 1$ integrins and then causes the translocation of R-Ras to early endosomes. Here, the R-Ras/RIN2/Rab5 signaling module activates Rac1-dependent cell adhesion via TIAM1, a Rac GEF that localizes on early endosomes and is stimulated by the interaction with both Ras proteins and the vesicular lipid phosphatidylinositol 3-monophosphate. In conclusion, the ability of R-Ras-GTP to convert RIN2 from a GEF to an adaptor that preferentially binds Rab5GTP allows the triggering of the endocytosis of ECM-bound/active $\beta 1$ integrins and the ensuing funneling of R-RasGTP toward early endosomes to elicit the pro-adhesive and TIAM1-mediated activation of Rac1.

Keywords: integrins; R-Ras; Rab5; endosomal signaling; angiogenesis

Cell Research (2012) 22:1479-1501. doi:10.1038/cr.2012.110; published online 24 July 2012

\section{Introduction}

In vertebrates, the appearance of vascular networks shaped to fulfill the disparate demands of nutrients by different tissues [1] and to optimize blood fluidodynamics [2] grounds on the possibility of modulating endothe-

*These three authors contributed equally to this work.

Correspondence: Guido Serini

Tel: +39 11 9933508; Fax: +39 119933524

E-mail: guido.serini@ircc.it

Received 26 August 2011; revised 6 May 2012; accepted 14 June 2012; published online 24 July 2012 lial cell (EC) adhesion, spreading, and motility. While the formation of a structurally and functionally normal vascular tree is essential for proper embryonic development [3], this usually does not apply to most of the malignant lesions that form in the adult organisms [4]. Indeed, solid cancers usually display an abnormal vasculature that impairs the optimal delivery of chemotherapy and oxygen, the latter being a pre-requisite for an effective radiotherapy [5]. Therefore, dissecting the molecular mechanisms that underpin the regulation of EC adhesion could allow a deeper understanding of both physiological and pathological vascular morphogenesis.

During the formation and remodeling of blood ves- 
sels, EC adhesion to provisional extracellular matrix (ECM) proteins, such as fibronectin (FN) and vitronectin (VN), is mediated by $\alpha 5$ and $\alpha \mathrm{v}$ integrin receptors [6, 7] that cooperate in remodeling the vasculature during angiogenesis [8]. Small GTPases belonging to the R-Ras branch of the wider Ras superfamily [9], namely R-Ras (also known as R-Ras1), TC21 (also known as R-Ras2), and M-Ras (also known as R-Ras3), have all been reported to enhance integrin-mediated cellto-ECM adhesion [10]. Of note, R-Ras expression is mainly restricted to ECs and vascular smooth muscle cells both in vivo and in vitro [11] and it has been implicated in cardiovascular diseases. Genome wide association studies identified a new susceptibility locus for coronary artery disease in the MRAS gene that is also highly expressed in the cardiovascular system [12]. In addition, the cytoplasmic domain of PlexinD1 receptor, which in ECs conveys signals elicited by several secreted chemorepellents belonging to the class 3 semaphorin family [13-15], functions as an R-Ras and M-Ras GTPase activating protein (GAP) [16]. Observations that genetic inactivation of plexinD1 in ECs causes severe cardiovascular defects in mouse embryos [15] and that PlexinD1 is overexpressed in the tumor vasculature of mouse models of cancer [17] further underscore the central role played by R-Ras GTPases and their regulators in both embryonic and tumor angiogenesis.

Our understanding of the molecular details through which R-Ras promotes EC adhesion to ECM proteins is however still patchy and incomplete. Investigating non-adherent myeloid cells and Chinese hamster ovary (CHO) cells overexpressing constitutively active R-Ras $38 \mathrm{~V}$, Zhang et al. [18] originally found that R-Ras signaling acts by increasing the affinity and avidity (i.e., clustering) of integrins. Regulation of the affinity of integrins for their ECM ligands largely depends on their conformational activation $[19,20]$; however, R-Ras $38 \mathrm{~V}$ does not directly stimulate integrin conformational activation in CHO cells $[10,21]$, thus suggesting that R-Ras should promote cell adhesion to the ECM by alternative mechanisms. For example, spreading and motile cells also control their adhesion dynamics by trafficking integrins [22-24] and recent evidence suggests how different molecular machineries and endosomal compartments govern the traffic of ligand-free/inactive and ECM-bound/active integrins [25-28]. Indeed, neuropilin-1 (Nrp1) membrane receptor, in association with the endocytic adaptor GIPC1/synectin, and the molecular motor myosin 6 selectively promote the endocytosis of FN-bound/active $\alpha 5 \beta 1$ integrin from fibrillar adhesions, thus regulating EC adhesion to FN and FN fibrillogenesis $[25,28]$. Once internalized into Rab5-positive early en- dosomes, ECM-bound/active $\alpha 5 \beta 1$ integrin accumulates in late endosomes $[26,27]$, where $\mathrm{pH}$ and the CLIC3 chloride channel control the recycling of ECM-freed integrins to the plasma membrane, which is mandatory for efficient cell migration [26].

Endocytosis and traffic of active integrins could support ECM adhesion dynamics either per se (see above) $[25,29]$ or by allowing the endosomal activation of signaling pathways, such as Src tyrosine kinase [26, 30] and/or Rho family GTPases [31, 32]. In spreading cells, the formation of active integrin-containing ECM adhesions is under the control of Rho GTPase-driven actin polymerization [33]. Depending on their size, shape, subcellular localization, molecular composition, and dynamics, ECM adhesive structures are classified as nascent adhesions (NAs), focal complexes (FCs), and focal adhesions (FAs) [34]. NAs are highly dynamic adhesive entities that appear in response to Rac activation as small dot-like structures at the periphery of spreading cells, where they associate with the loose peripheral actin meshwork [35]. In response to RhoA-elicited actomyosin contractility, NAs mature first into larger and round FCs, located at the lamellipodium-lamellum interface, and then into long FAs, located at the end of the actin stress fibers and displaying a slower turnover than NAs [34]. In this framework, hence, the ability of R-Ras to trigger the activation [36, 37] and ensuing Arf6-driven translocation of Rac-GTP to the cell surface [38], where it can then promote actin polymerization, cell adhesion, and spreading, is of particular relevance [39]. In the pathway that controls the spatially restricted activation of Rac, in addition to R-Ras, the small GTPase Rab5, a crucial regulator of the early steps of endocytosis [40], has also been found to play a relevant function $[31,41]$. Indeed, Rab5-positive early endosomes (EE) can act as signaling platforms on which Rac is first GTP-loaded by the phosphatidylinositol 3-monophosphate (PIns(3)P)- and Ras-regulated guanyl exchange factor (GEF) T-lymphoma invasion and metastasis-inducing protein 1 (TIAM1) [42-45] and then recycled to the plasma membrane in an Arf6-dependent manner [31] to induce cell migration.

Here, we identify the Ras and Rab interactor 2 (RIN2) protein as a key R-Ras mediator that, by physically and functionally coupling R-Ras and Rab5 GTPases at NAs and on early endosomes, elicits EC-to-ECM adhesion, migration, and vascular morphogenesis. Upon cell binding to the ECM, the association of RIN2 with R-RasGTP lessens its Rab5 GEF activity and maximizes its docking function. As an adaptor protein, on the one hand RIN2 concentrates a pool of Rab5 at NAs, while on the other hand it promotes the Rab5-dependent topological relocation of active R-Ras to Rac1-containing early 
endosomes. Accordingly, R-Ras-GTP via RIN2/Rab5 specifically elicits the endocytosis of ECM-bound/ active integrins from the plasma membrane while the concurrent association of RIN2 with active R-Ras and Rab5, which at steady state mainly occurs on EEA1positive early endosomes, is instead required to stimulate the activation of Rac1 and the ensuing adhesion of ECs to ECM proteins. Furthermore, stimulation of cell adhesion by RIN2 depends on TIAM1, a Rac GEF that can be activated on early endosomes by the coincident detection of two Rab5-triggered phenomena: the VPS34-mediated production of PIns(3)P and the RIN2dependent translocation of R-Ras-GTP.

\section{Results}

$R$-Ras interacts with the Rab5-binding protein RIN2 to stimulate EC adhesion

Overexpression of wild-type (WT) or constitutively active R-Ras $38 \mathrm{~V}$ small GTPase promotes the adhesion of different cell types to ECM proteins [10]. However, despite its restricted tissue distribution to ECs and vascular smooth muscle cells [11], a direct evidence of the role of endogenous R-Ras in the regulation of EC adhesion is still lacking. To investigate this, we silenced R-Ras in human umbilical vein ECs by transducing them with three different lentivirus-delivered short hairpin RNA (shRNA). Western blot analysis revealed that, in comparison with control cells (shCtl), R-Ras protein, but not $\beta$-tubulin, was successfully silenced in shhR-Ras ECs (Figure 1A). Next, we examined the effect of R-Ras silencing on EC adhesion to ECM proteins. Short-term adhesion assays showed that loss of R-Ras significantly reduced EC adhesion to FN and VN (Figure 1B). Similarly, R-Ras silencing impaired EC adhesion to type I collagen (Coll I) (Supplementary information, Figure S1A). Thus, endogenous R-Ras is a general positive regulator of EC-to$\mathrm{ECM}$ adhesion.

To investigate the underlying mechanisms of R-Rasinduced EC adhesion to the ECM, we performed a yeasttwo-hybrid screen employing constitutively active R-Ras $38 \mathrm{~V}$ as bait. Interestingly, in addition to confirming that Ral GEFs are major R-Ras-associated proteins [46, 47], we found that another traffic regulator, namely the Rab5binding protein RIN2, accounted for about one fourth of the isolated preys (Supplementary information, Table S1). Thus, we were interested to study whether RIN2 could be a major effector of the R-Ras pro-adhesive activity in ECs. Together with RIN1 and RIN3, RIN2 belongs to the RIN family of Rab5 GEFs [48]. While RIN1 and RIN3 protein synthesis is highly restricted to nervous and hematopoietic tissues, respectively, RIN2 is instead more broadly expressed [49].

To understand whether the association of RIN2 was sensitive to the conformational activation of R-Ras, we verified whether RIN2 could interact with either constitutively active $(38 \mathrm{~V})$ or dominant-negative $(43 \mathrm{~N})$ $\mathrm{R}-\mathrm{Ras}$ in mammalian cells. Therefore, we generated an N-terminal hemagglutinin (HA)-tagged full-length murine RIN2 construct ( $m$ RIN2) and co-transfected it in NIH-3T3 fibroblasts together with either green fluorescent protein (GFP), or GFP-tagged constitutively active R-Ras $38 \mathrm{~V}$, or dominant-negative R-Ras 43N. Cell lysates were then immunoprecipitated with anti-GFP and blotted with an anti-HA antibody (Supplementary information, Figure S2A). We found that RIN2 efficiently associated with GFP-tagged active R-Ras $38 \mathrm{~V}$, but neither with its dominant-negative mutant (R-Ras $43 \mathrm{~N}$ ) nor with GFP alone. Moreover, we showed that, in NIH3 T3 cells co-transfected with HA-tagged $m$ RIN2 and GFP-tagged Rab5A or Rab7, RIN2 is able to bind the early endocytic small GTPase Rab5A, but not the late endosome/lysosome small GTPase Rab7 (Supplementary information, Figure S2B). Therefore, as inferred from the yeast two-hybrid system and co-immunoprecipitation analysis in mammalian cells, RIN2 protein associates with both active R-Ras and Rab5A.

Next, we investigated whether RIN2 could participate in the signaling pathways that control EC adhesion to the ECM. We oligofected human ECs with either a pool of four siRNAs targeting human RIN2 (sihRIN2) or control non-targeting siRNA ( siCtl). Western blot analysis confirmed that RIN2 protein, but not $\beta$-tubulin, was effectively silenced in sihRIN2 ECs compared to control cells (Figure 1C). Short-term adhesion assays revealed that loss of RIN2 significantly impaired EC adhesion to FN and VN (Figure 1D). In addition, upon retrovirus-mediated $m$ RIN2 overexpression the number of ECs adhering on both ECM proteins was significantly higher than that of control-transduced cells (see below). Since RIN2 clearly behaved as a positive regulator of EC-to-ECM adhesion, we then tested whether RIN2 was required for the induction of cell adhesion by R-Ras. As illustrated in Figure 1E, silencing of human RIN2 with shRNA fully abrogated or severely impaired the ability of WT and constitutively active R-Ras $38 \mathrm{~V}$ to promote $\mathrm{EC}$ adhesion to $\mathrm{FN}$ and VN. Likewise, RIN2 regulated both basal and R-Ras 38V-elicited adhesion of ECs to Coll I as well (Supplementary information, Figure S1B). In addition, FACS analysis revealed that both R-Ras and RIN2 silencing inhibit to a similar extent rhodamine-FN binding to ECs (Figure 1F). Collectively, these results indicate that the Rab5-binding protein RIN2 is a key mediator of R-Ras-elicited EC adhesion to the ECM. 
A

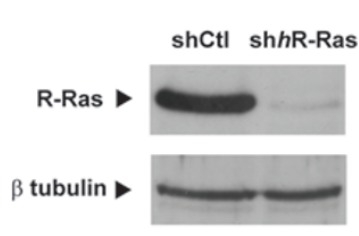

E

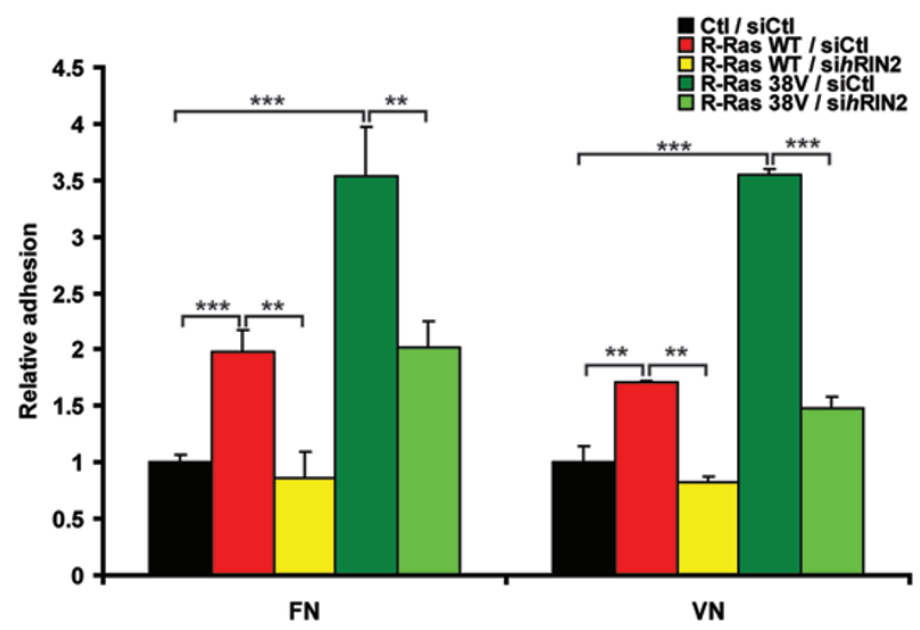

B

C

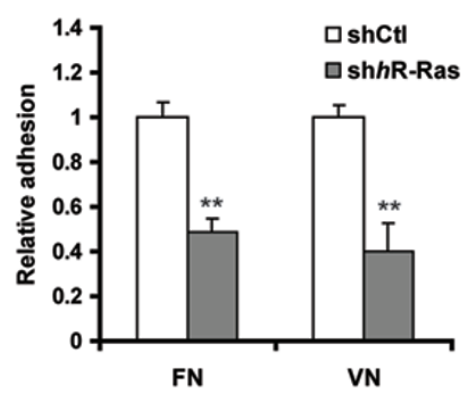

D

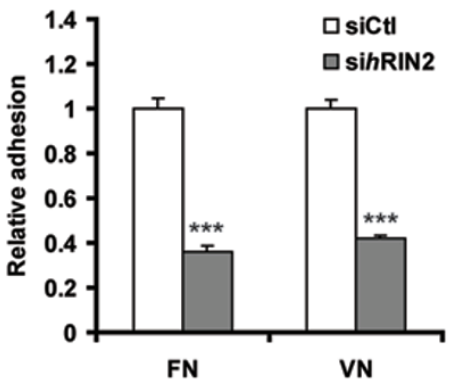

F

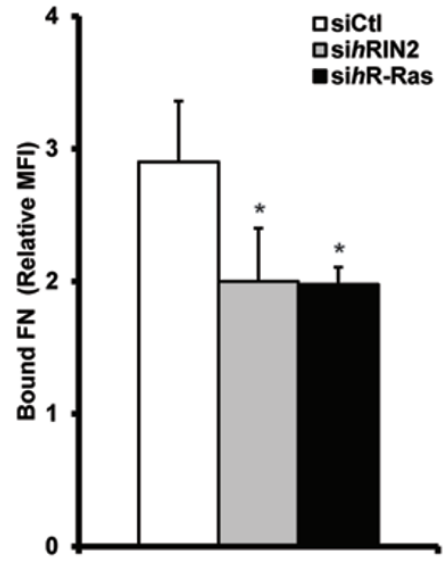

G

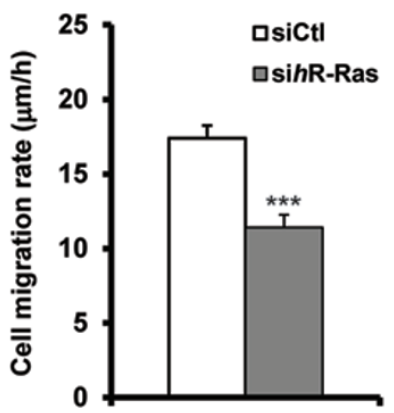

H

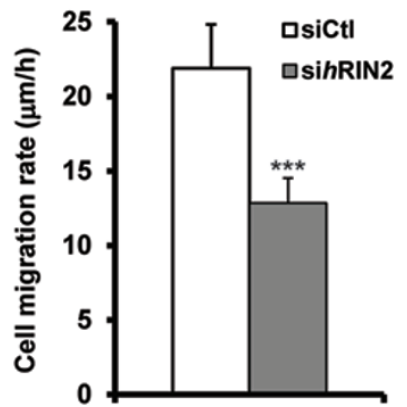

I

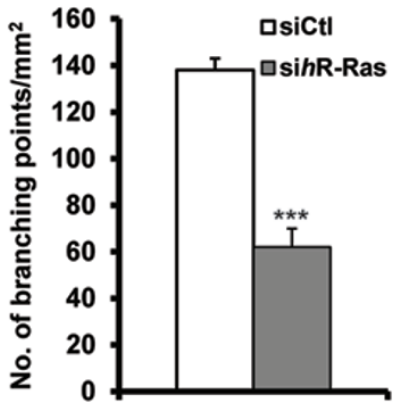

L

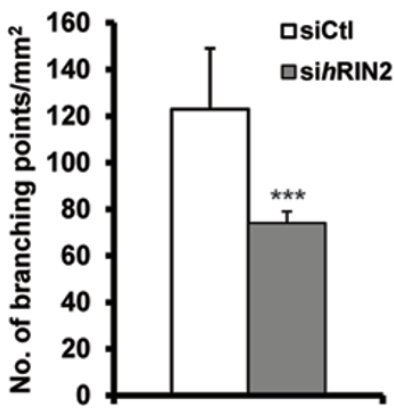

M

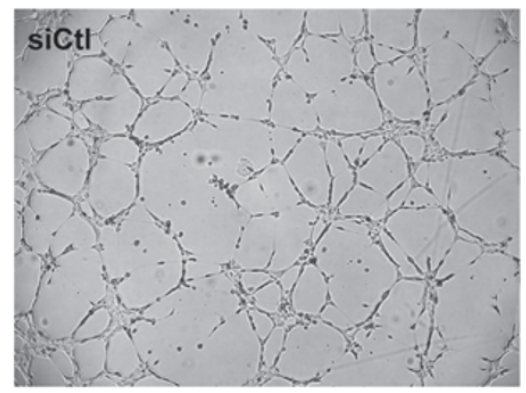

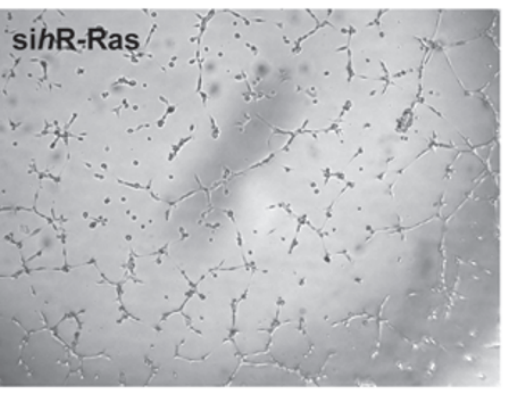

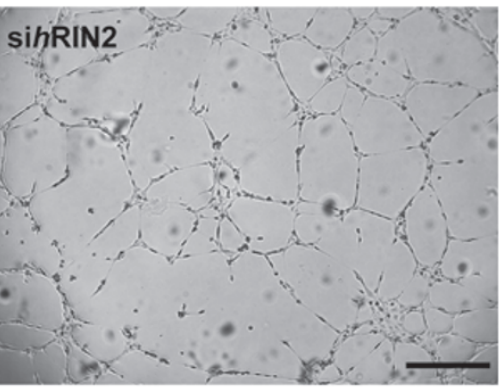


During embryonic development and tumor angiogenesis, vascular morphogenesis relies on the ability of ECs to move and dynamically control their integrindependent adhesive interactions with the surrounding ECM $[6,7,50]$. Therefore, we first investigated whether and how R-Ras and RIN2 protein expression could influence motility of ECs on their own endogenous ECM, mostly constituted by FN [25], as measured in wound healing assays captured by time-lapse phasecontrast videomicroscopy [51]. Both R-Ras (Figure 1G and Supplementary information, Movie S1) and RIN2 (Figure $1 \mathrm{H}$ and Supplementary information, Movie S2) silencing resulted in a significant reduction of EC migration rate. Next, we studied vascular morphogenesis in vitro by exploiting the permissive activity of Matrigel, a natural laminin-containing basal membrane matrix that unleashes the cell autonomous property of cultured ECs to self-aggregate and morphologically differentiate in tubular networks almost identical to those observed in vivo [52]. In this morphogenetic assay, vascular networks resulting from the aggregation of both $\operatorname{si} h \mathrm{R}$ Ras (Figure 1I and $1 \mathrm{M}$ ) and sihRIN2 (Figure 1L and $1 \mathrm{M}$ ) ECs plated on Matrigel were disrupted and significantly less branched when compared to those formed by siCtl ECs. Thus, R-Ras and RIN2 control the ability of ECs to adhere, directionally migrate, and self-organize on ECM substrates, finally giving rise to properly organized vascular networks.

R-Ras and RIN2 localize with Rab5 both at nascent adhesions and on early endosomes

To gain insight into mechanisms by which R-Ras and RIN2 could cooperate in regulating EC adhesiveness, we characterized by fluorescent confocal microscopy their subcellular localizations in ECs spreading on FN. We found that in cultured ECs, $m$ RIN2 colocalized with GFP-R-Ras $38 \mathrm{~V}$ in dot-shaped NAs located at the cell borders (Figure 2A, arrowheads) and in vesicular structures (Figure 2A, dashed arrows). We identified the vesicles on which RIN2 was targeted together with R-Ras 38V (Figure 2A, dashed arrows) as GFP-Rab5A-positive early endosomes (Figure 2C). Accordingly, as shown in Supplementary information, Figure S3, $m$ RIN2 and R-Ras $38 \mathrm{~V}$ double-positive endosomes also contained the early endosome marker EEA1, but neither LAMP1 nor TGN46, which label late endosomes/lysosomes and trans-Golgi network, respectively. In addition, GFP-Rab5A colocalized with $m$ RIN2 in NAs (Figure 2C). Further analysis showed that upon co-transfection in ECs, $m$ RIN2 fully colocalized with GFP-vinculin-containing NAs in the outermost region of the cell periphery (Figure $2 \mathrm{~B}$, external arrowheads), while in the innermost area (Figure 2B, internal arrowheads) $m$ RIN2 overlapped only with the external portion of vinculin-labeled FCs that were maturing into FAs, from which $m$ RIN2 was excluded (Figure 2B, arrows and Supplementary information, Figure S4). To further confirm the localization of R-Ras and RIN2 in NAs, we treated ECs with blebbistatin, a myosin II inhibitor that blocks the force-dependent maturation of NAs into FCs and hence maximizes the formation of NAs $[35,53]$. In blebbistatin-treated ECs R-Ras $38 \mathrm{~V}$ and $m$ RIN2 clearly localized not only in vesicular structures, but also in vinculin-positive NAs that accumulated in lamellipodia at the cell periphery because of the inhibition of myosin II-dependent contractility that drives the transformation of NAs into FCs and FAs

Figure 1 R-Ras and RIN2 promote EC-to-ECM binding, adhesion, migration, and vascular morphogenesis; RIN2 mediates the pro-adhesive effects of R-Ras. (A) Representative western blot analysis of R-Ras and $\beta$-tubulin protein expression in control (shCtl) and R-Ras-silenced (shhR-Ras) human ECs. (B) Comparison between shCtl and shhR-Ras ECs adhering to FN and VN. A representative of three independent experiments with similar results is shown (mean \pm SD; $n=3$ samples per experimental condition). (C) Western blot analysis of RIN2 expression in human ECs silenced for RIN2 (sihRIN2) or transfected with control siRNA (siCtl). $\beta$-tubulin was used as an internal and loading control. (D) Comparison between siCtl- and sihRIN2transfected human ECs adhering to FN and VN. A representative of eight (FN) and five (VN) independent experiments with similar results is shown (mean $\pm \mathrm{SD} ; n=3$ samples per experimental condition). (E) Comparative analysis of stimulation of cell adhesion to FN and VN by WT and constitutively active R-Ras 38V in either control (shCtl) or RIN2-silenced (shhRIN2) human ECs. Ctl corresponds to ECs transduced with the empty PINCO retroviral vector. A representative of three independent experiments with similar results is shown (mean $\pm \mathrm{SD} ; n=3$ samples per experimental condition). (F) FACS analysis of rhodamine-FN binding to siCtl, sihR-Ras, and sihRIN2 ECs. A representative of three independent experiments with similar results is shown (mean $\pm \mathrm{SD} ; n=4$ samples per experimental condition). (G, H) Comparison between siCtl and sihR-Ras (G) or sihRIN2 $(\mathbf{H})$ EC migration rate $(\mu \mathrm{m} / \mathrm{h})$ in wound healing assays. A representative of three independent experiments with similar results is shown (mean $\pm \mathrm{SD} ; n=3$ samples per experimental condition). (I-L) Comparison of the number of branching points $/ \mathrm{mm}^{2}$ of vascular networks formed by siCtl and sihR-Ras (I) or sihRIN2 (L) ECs plated on growth factor-reduced Matrigel matrix. A representative of three independent experiments with similar results is shown (mean $\pm \mathrm{SD} ; n=3$ samples per experimental condition). (M) Representative pictures of vascular networks formed by siCtl, sihR-Ras, sihRIN2 ECs plated on growth factor-reduced Matrigel matrix. Scale bars, $500 \mu \mathrm{m}$. ${ }^{*} P<0.05,{ }^{* \star} P<0.01,{ }^{* * *} P<0.001$. 
A
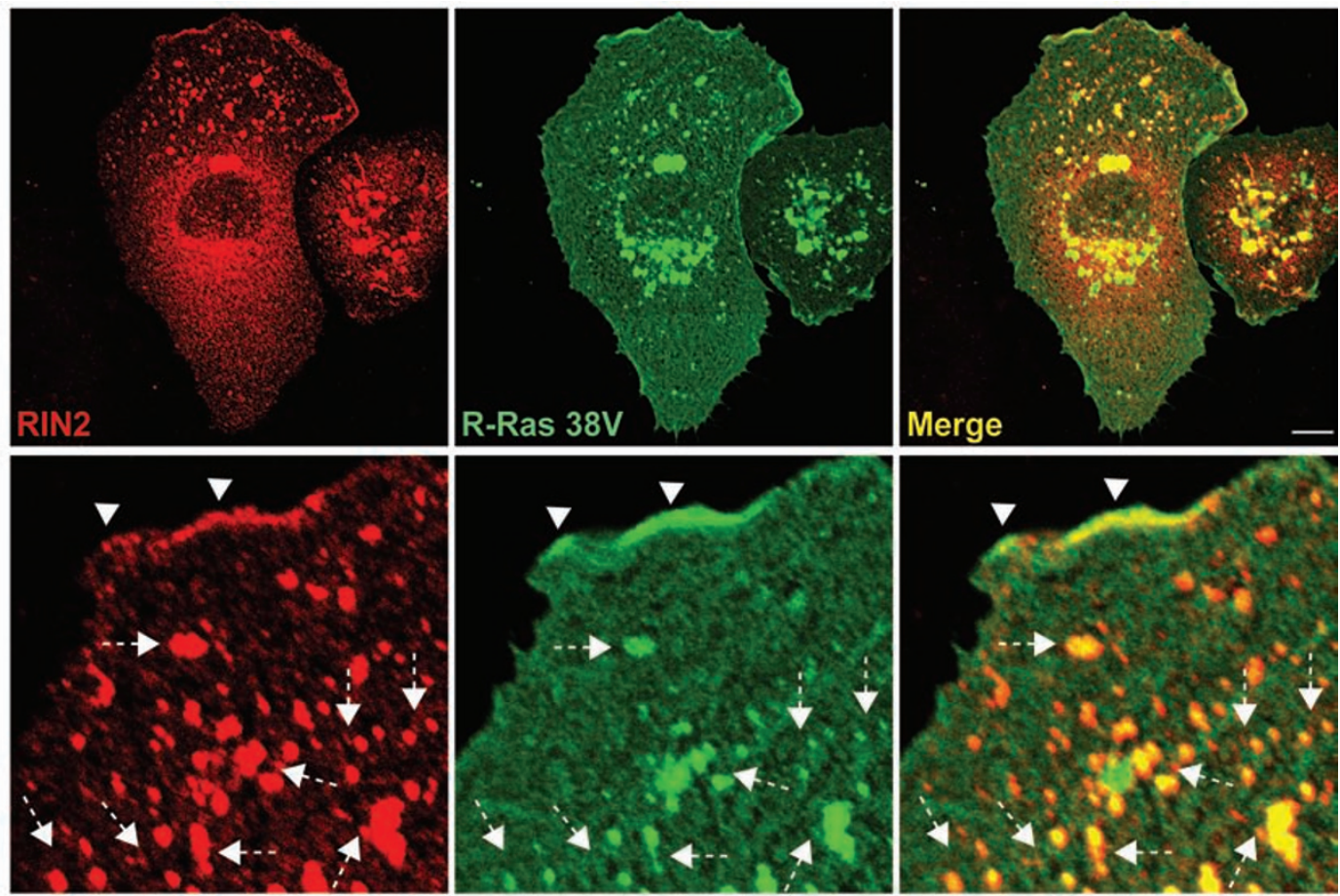

B
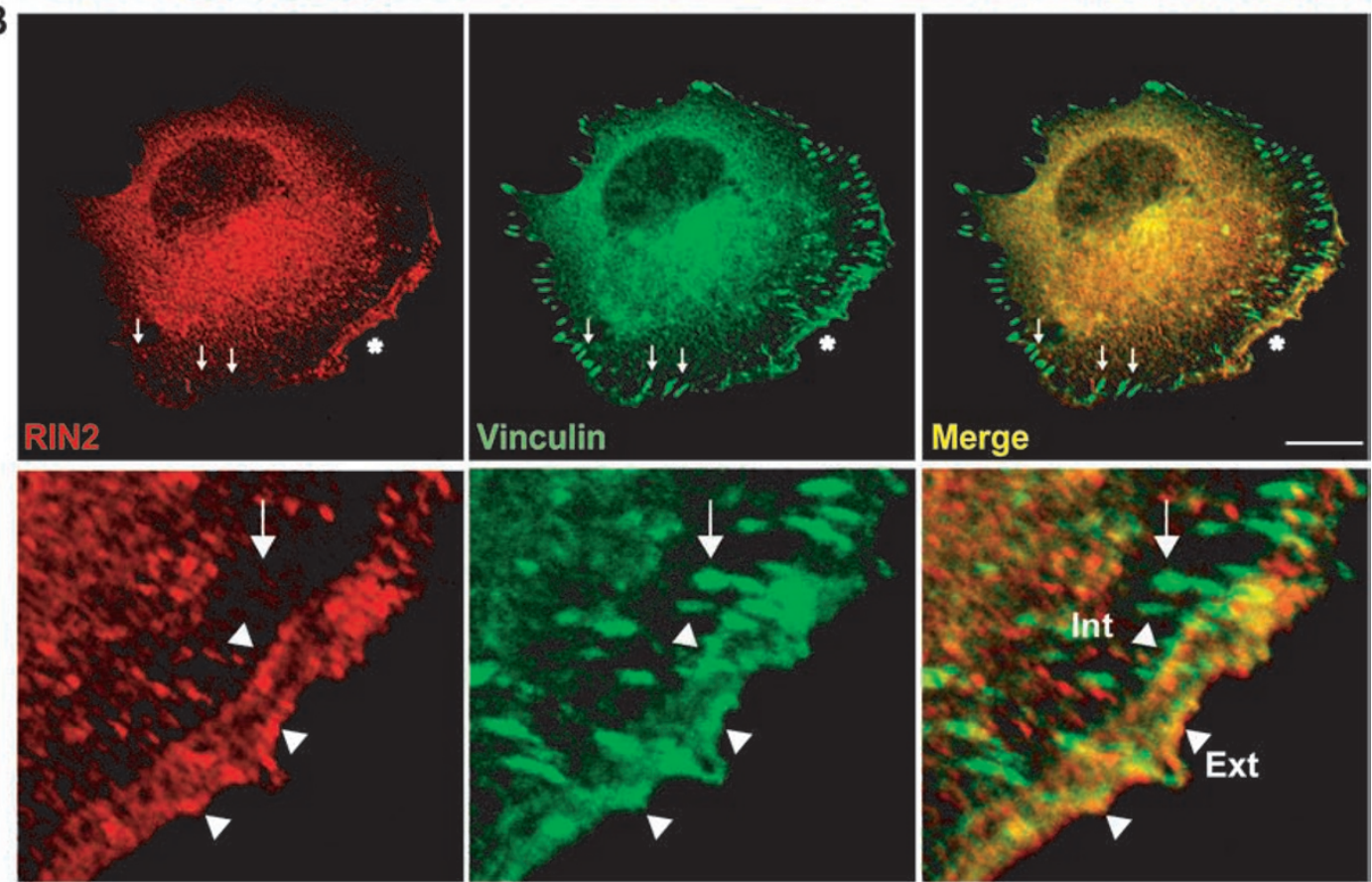

C

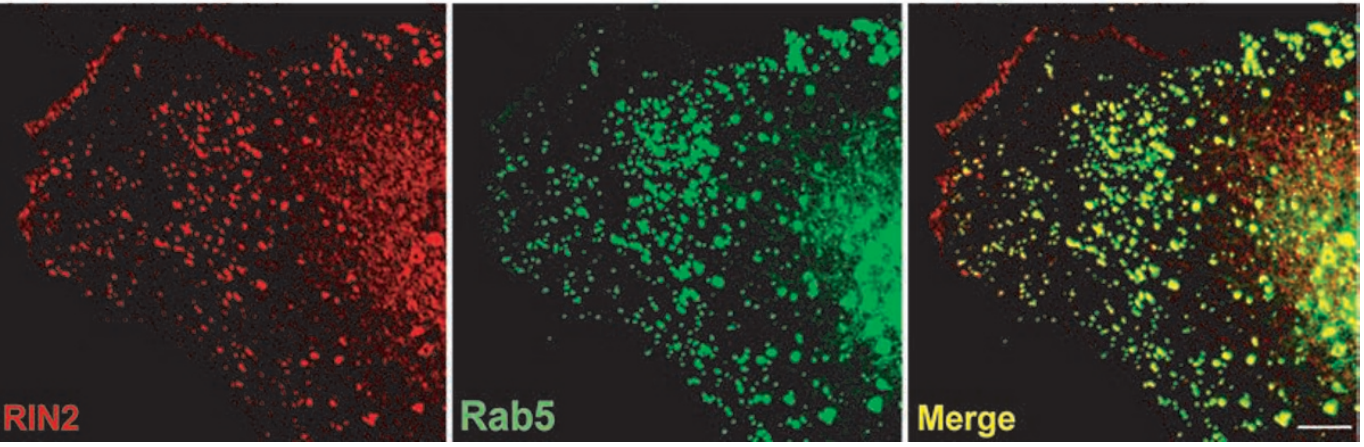

Cell Research | Vol 22 No 10 | October 2012 
(Supplementary information, Figure S5). Remarkably, the amount of GFP-Rab5 that colocalized with $m$ RIN2 at NAs became more evident after blebbistatin treatment (Supplementary information, Figure S5). Thus, to initiate signals that regulate EC adhesion and spreading, RRas, RIN2, and Rab5 meet in a myosin II-driven way at lamellipodia-associated NAs and on early endosomes.

\section{Reciprocal control of R-Ras/RIN2 and ECM-bound/ac- tive $\beta 1$ integrin endosomal traffic}

In cells migrating over the ECM, adhesion dynamics can be modulated by regulating integrin conformational activation, traffic, or both [24]. Therefore, by capture ELISA upon biotin labeling of surface proteins we initially compared the amount of total (i.e., mostly ligandfree/inactive) and ECM-bound/active $\beta 1$ integrins, as revealed by the $9 \mathrm{EG} 7 \mathrm{mAb}$ [54], on the plasma membrane of siCtl, si $h$ R-Ras, and si $h$ RIN2 ECs. In accordance with the study by Sehti et al. [21] that overexpressed constitutively active R-Ras in CHO cells, we found that in ECs neither R-Ras (Figure 3A and 3B) nor RIN2 (Figure 3C and $3 \mathrm{D}$ ) silencing affected the surface amount of either total (Figure 3A and 3C) or active (Figure 3B and 3D) $\beta 1$ integrins compared to control cells, thus indicating that R-Ras should regulate EC-to-ECM adhesion through mechanisms alternative to the control of integrin conformational activation.

Since we found that RIN2 could localize together with its interactors R-Ras and Rab5 both at NAs and on early endosomes and previous overexpression studies suggested that R-Ras can influence integrin traffic [55], we biochemically quantified the effect of R-Ras or RIN2 silencing on total and active integrin endocytosis. Notably, although internalization of total $\beta 1$ integrin (Figure 3E and $3 \mathrm{G}$ ) was not significantly impaired in either sihR-Ras (Figure 3E) or sihRIN2 (Figure 3G) ECs, knockdown of either R-Ras (Figure 3F) or RIN2 (Figure 3H) selectively reduced the amount of active (9EG7-positive) $\beta 1$ integrin endocytosed by ECs. Furthermore, we found (Figure 3I) that overexpressing either WT or constitutively ac- tive R-Ras $38 \mathrm{~V}$ caused a dramatic increase of active $\beta 1$ integrin internalization that depended on RIN2. Finally, since RIN2 is a Rab5 GEF [48, 56], we simultaneously silenced in ECs the three Rab5 isoforms Rab5A, Rab5B, and $\mathrm{Rab} 5 \mathrm{C}$ and, as expected, we detected a significant decrease of both total and active $\beta 1$ integrin endocytosis (Supplementary information, Figure S6). Hence, analogously to what we already observed for Nrp1-GIPC1/ synectin-Myo6 complex [25], in ECs R-Ras and RIN2 localize at ECM adhesion sites, where active integrin receptors concentrate, and, likely at this location, they promote the Rab5-dependent endocytosis of ECM-bound/ active $\beta 1$ integrins.

ECM-bound/active integrin internalization could favor cell spreading via at least two non-mutually exclusive mechanisms, i.e., by supporting adhesion site dynamics or by triggering pro-adhesive signals from endosomal platforms [25, 26, 28, 57]. Therefore, we analyzed the endosomal localization of HA-mRIN2, Rab5-GFP, and WT or constitutively active monomeric red fluorescent protein (mCherry)-R-Ras $38 \mathrm{~V}$ in adherent or non-adherent suspended ECs (Supplementary information, Figure S7). Notably, we found that while constitutively active mCherry-R-Ras 38V localized on RIN2- and Rab5-positive early endosomes both in adherent and non-adherent ECs, the same was not true for WT mCherry-R-Ras. Indeed, keeping ECs in suspension severely impaired the localization of both WT mCherry-R-Ras and $m$ RIN2 on the Rab5 vesicular compartment (Supplementary information, Figure S7). However, likely because ECM binding to integrins triggers GTP loading of R-Ras [58, 59], addition of soluble FN significantly increased the relocation of both WT mCherry-R-Ras and $m$ RIN2 to Rab5 endosomes of suspended ECs (Supplementary information, Figure S7). Thus, our data suggest a model in which ECM binding to integrins triggers the GTP loading of R-Ras at NAs and its binding to RIN2, which via Rab5-GTP in turn favors the endocytosis of ECM-bound/ active integrins and simultaneously relocates R-Ras-GTP to early endosomes.

Figure 2 RIN2 colocalizes with active R-Ras in NAs, FCs and Rab5-positive early endosomes. (A) Fluorescent confocal microscopy analysis of ECs transfected with $m$ RIN2 (left panels, red) and GFP-R-Ras 38V (central panels, green) indicates that $m R I N 2$ and GFP-R-Ras 38V colocalize in peripheral NAs (arrowheads), and vesicles (dashed arrows) as shown in merge (right panels). Lower panels are magnifications of the corresponding upper panels. (B) Fluorescent confocal microcopy reveals that in ECs transfected with $m$ RIN2 (left panels, red) and GFP-vinculin (central panels, green), $m$ RIN2 colocalizes (right panels) in full with peripheral NAs (external arrowheads), in part with the external portion of FCs maturing into FAs (internal arrowheads), but not with elongated FAs (arrows). Lower panels are magnifications of the corresponding upper panels. (C) Fluorescent confocal analysis of ECs transfected with mRIN2 (left panels, red) and GFP-Rab5A (central panels, green) unveils that $m$ RIN2 and Rab5A colocalize in early endosomes as shown in merge (right panels). A minor amount of GFP-Rab5A is present at the cell periphery as well, where it colocalizes with HA-RIN2 in NAs. Panels are representative of more than 25 cells in four independent experiments. Scale bars, $10 \mu \mathrm{m}$. 

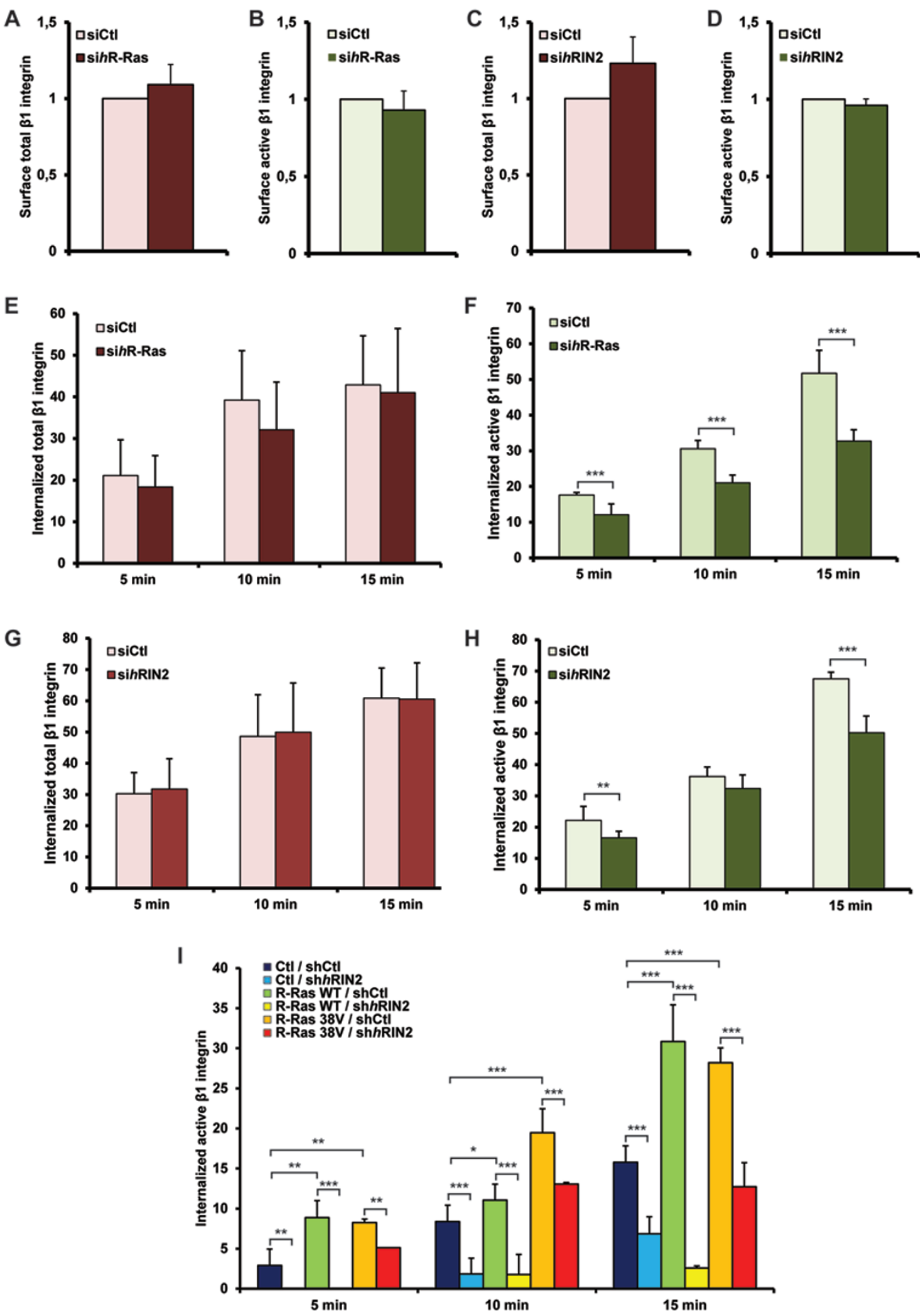

Figure 3 R-Ras controls active $\beta 1$ integrin endocytosis via RIN2 in ECs. (A-D) Comparison of the amounts of total (A, C) or active (B, D) $\beta 1$ integrins on the plasma membrane of siCtl versus sihR-Ras (A, B) or sihRIN2 (C, D), as evaluated by surface biotinylation and capture ELISA assays. Values are mean \pm SD from more than three independent experiments $(n=6$; $P \geq 0.2$ and differences are not statistically significant). (E-H) Time-course analysis of the amounts of total $(\mathbf{E}, \mathbf{G})$ or active $(\mathbf{F}$, H) $\beta 1$ integrins endocytosed in siCtl versus sihR-Ras (E, F) or sihRIN2 (G, H), as evaluated by integrin internalization assay and capture ELISA assay. Values are mean \pm SD from more three independent experiments $(n=6)$. In $\mathbf{E}$ and $\mathbf{G}, P \geq 0.3$ and differences are not statistically significant. (I) Time-course analysis of the amounts of active $\beta 1$ integrins endocytosed in shCtl versus shhRIN2 ECs overexpressing or not R-Ras WT or $38 \mathrm{~V}$, as evaluated by integrin internalization assay and capture ELISA assay. Values are mean \pm SD from more than three independent experiments $(n=6) .{ }^{*} P<0.05,{ }^{* *} P<0.01,{ }^{* *} P<0.001$. 
RIN2 regulates the adhesion of ECs to ECM proteins via its Rab5 and Ras binding domains

Proceeding from the $\mathrm{N}$ - to the $\mathrm{C}$-terminus, $\mathrm{RIN}$ proteins display a phosphotyrosine-binding Src-homology-2 (SH2) domain followed by one or more proline-rich domains (PRDs), a RIN-homology (RH) domain that together with the GEF domain VPS9 is responsible for binding to Rab5 GTPase, and a Ras-associating (RA) domain that mediates the interaction of RIN proteins with Ras GTPases (Figure 4A). All together, the above results suggest that the stimulation of EC cell adhesion by RIN2 could depend on its ability to bring active R-Ras onto the Rab5-containing early endosomal compartment. To address this issue, we first generated two HA-tagged deletion mutants of $m$ RIN2 lacking either the RH and VPS9 domains ( $m$ RIN2 $\triangle \mathrm{RH} / \mathrm{VPS} 9)$, which are responsible for RIN2 binding to and GTP loading of Rab5, or the RA domain ( $m$ RIN2 $\triangle \mathrm{RA}$ ) that could mediate the interaction between RIN2 and active R-Ras (Figure 4A). As expected, biochemical analysis showed that compared to WT $m$ RIN2, $m$ RIN2 $\Delta$ RH/VPS9 and $m$ RIN2 $\Delta$ RA mutant proteins were unable to bind active Rab5 (Supplementary information, Figure S8) and R-Ras (Supplementary information, Figure S9) GTPases, respectively. Then, we compared the ability of retrovirus-delivered full-length and deletion constructs of $m$ RIN2 to rescue the adhesion defects of human ECs in which RIN2 had been silenced by a lentiviral shRNA ( $\operatorname{sh} h$ RIN2) that did not target $m$ RIN2 constructs (Figure 4B). In comparison to the WT $m$ RIN2, both $m$ RIN2 $\Delta$ RH/VPS9 and $m$ RIN2 $\Delta$ RA constructs were fully impaired in their ability to rescue the adhesion defects of sh $h$ RIN2 ECs on FN and VN (Figure 4C). Furthermore, while the overexpression of WT $m \mathrm{RIN} 2$ stimulated the adhesion of ECs to ECM proteins, both $m$ RIN2 $\Delta$ RH/VPS9 and $m$ RIN2 $\Delta$ RA displayed a dominant-negative effect, with a strong reduction of the number of cells adhering to ECM proteins (Figure 4D).

To get further insight into the dominant-negative activity of $m$ RIN2 $\Delta$ RH/VPS9 and $m$ RIN2 $\Delta$ RA deletion mutants, we compared their subcellular localization with that of WT $m$ RIN2 in ECs where they were overexpressed together with mCherry-R-Ras $38 \mathrm{~V}$ and GFPRab5A. In agreement with biochemical evidence that in ECs endogenous R-Ras, RIN2, and Rab5 simultaneously interact (Figure 4E), confocal microscopy unveiled how a significant fraction of HA-tagged WT $m$ RIN2 (Figure 5A and 5D) colocalized with both mCherry-R-Ras $38 \mathrm{~V}$ and GFP-Rab5A on the same early endosomes. Conversely, $m$ RIN2 $\Delta$ RH/VPS9 localized mostly on mCherry-RRas 38V-positive vesicles, but significantly less on GFPRab5A-positive endosomes (Figure 5B and 5D). Vice versa, most of $m$ RIN2 $\triangle \mathrm{RA}$ was present on GFP-Rab5- positive vesicles, but almost absent from mCherry-R-Ras $38 \mathrm{~V}$-positive endosomes (Figure 5C and 5D). Hence, the pro-adhesive function of RIN2 clearly correlates with its ability to simultaneously associate on early endosomes with active Rab5 and R-Ras small GTPases via its RH/ VPS9 and RA domains, respectively. Accordingly, in addition to impairing EC adhesion, a major effect of dominant-negative $m$ RIN2 $\Delta$ RH/VPS9 and $m$ RIN2 $\Delta$ RA deletion mutants is the abrogation of the colocalization of R-Ras, RIN2, and Rab5 on early endosomes.

\section{The Rab5 GEF RIN2 acquires Rab5 effector properties upon R-Ras, but not H-Ras binding}

Our finding that RIN2 needs to simultaneously bind R-Ras and Rab5 to elicit the adhesion of ECs to the ECM prompted us to study the functional relationships between R-Ras, RIN2, and Rab5 in regulating the adhesive interactions among ECs and ECM molecules.

RIN proteins, via their VPS9 domain, function as Rab5 GEFs [9]. It has been shown previously that the binding of active H-Ras to the RBD domain of RIN1 stimulates its Rab5 GEF activity [60] and, at least in some cell types, H-Ras has also been found to counteract the pro-adhesive activity of R-Ras [10]. Notably, when we assayed the ability of purified RIN2 protein to stimulate the nucleotide exchange from unlabeled GDP to $\left[{ }^{3} \mathrm{H}\right]$-GDP on Rab5A, we observed that GTP $\gamma$ S-loaded HRas and R-Ras behaved differently. In particular, R-Ras-, but not H-Ras-GTP $\gamma \mathrm{S}$ (compared to Rab5 + $m$ RIN2 alone) reduced the rate of RIN2-catalyzed nucleotide exchange of Rab5A (Figure 6A). Moreover, extending previous in vitro association studies on RIN2 $[48,56]$ and RIN3 [61], we found that, unlike RIN1 [60], GTP $\gamma$ Sloaded glutathione S-transferase (GST)-Rab5A pulled down $m$ RIN2 from lysates of R-Ras-expressing NIH-3T3 cells much more efficiently than its GDP-loaded counterpart (Supplementary information, Figure S8). Both findings strongly indicate that R-Ras-bound RIN2 acts rather as a Rab5 effector or docker and only a slow acting GEF protein. Therefore, active R-Ras, by binding and switching the prevailing function of RIN2 from Rab5 GEF to adaptor protein, could first cause the recruitment of a pool of Rab5 at NAs (Supplementary information, Figure S5) and then warrant its own stable relocation to Rab5containing early endosomes (Figure 5).

\section{An R-Ras/RIN2/Rab5 signaling module controls EC ad- hesion to the ECM}

The latter observation, together with the fact that when overexpressed, Rab5 [62], similarly to R-Ras and RIN2 (see above), increases integrin-dependent cell adhesion to the ECM, suggested that the pro-adhesive activity of 
A

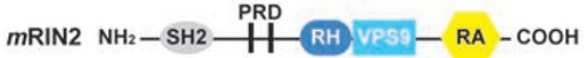

B

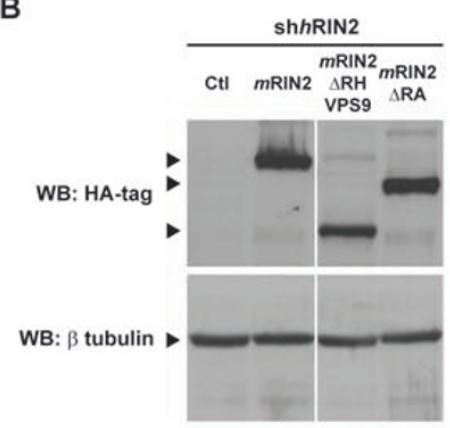

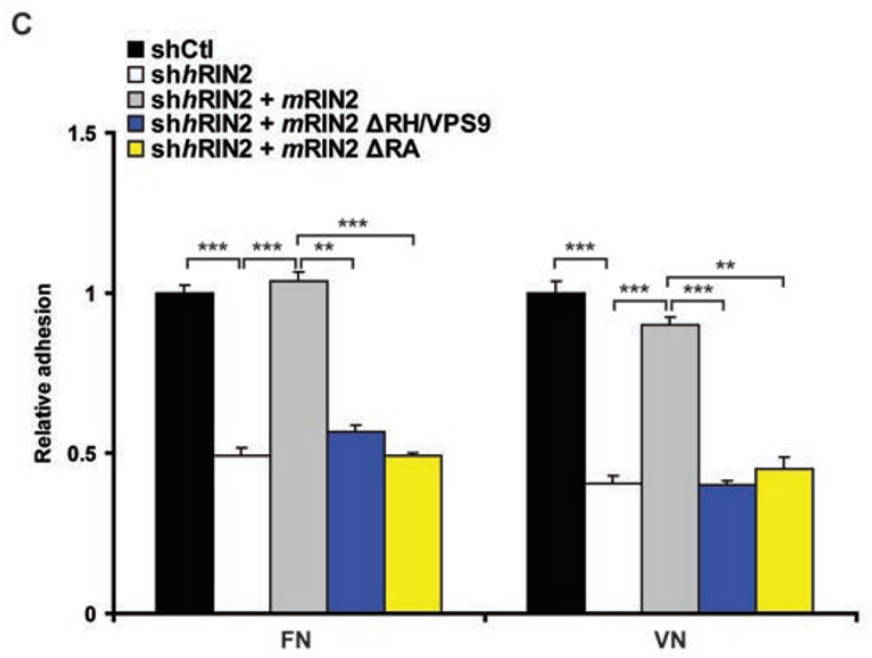

D
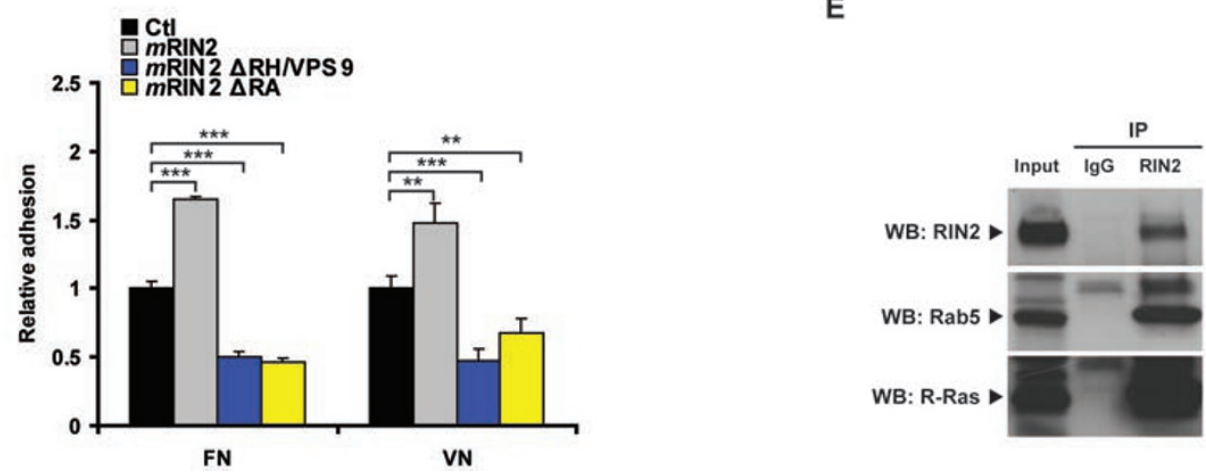

Figure 4 Stimulation of EC adhesion to ECM proteins by RIN2 depends on its Rab5- and Ras-binding domains and endogenous RIN2 associates with both R-Ras and Rab5. (A) Schematic representation of full-length $m$ RIN2 together with its Rab5and Ras-binding defective deletion mutants $m R I N 2 \Delta R H / V P S 9$ and $m R I N 2 \Delta R A$. (B) Western blot analysis of exogenously transduced and silencing-resistant HA-tagged $m R$ IN2 constructs and endogenous $\beta$-tubulin expression in ECs first infected with PINCO retrovirus carrying or not (Ctl) $m$ RIN2 constructs and then with a shhRIN2 lentivirus. (C) Comparison of WT fulllength $m$ RIN2, $m R I N 2 \Delta R H / V P S 9$, and $m R I N 2 \Delta R A$ efficiency in rescuing the defective adhesion of sh $h$ RIN2 ECs to FN and VN. A representative of three independent experiments with similar results is shown (mean \pm SD; $n=3$ samples per experimental condition). (D) Comparative analysis of the modulation of cell adhesion to FN and VN by overexpression of either fulllength $m$ RIN2 or $m$ RIN2 $\Delta$ RH/VPS9 and $m$ RIN2 $\Delta$ RA deletion mutants in human ECs. Ctl corresponds to ECs transduced with the empty PINCO retroviral vector. A representative of three independent experiments with similar results is shown (mean $\pm \mathrm{SD} ; n=3$ samples per experimental condition). (E) Immunoprecipitation (IP) of endogenous $h$ RIN2 followed by western blotting with anti-R-Ras, anti-Rab5, or anti-RIN2 antibodies. In ECs, RIN2 associates with both R-Ras and Rab5. An EC lysate was employed as positive control or immunoprecipitated with irrelevant IgG for negative control purposes. ${ }^{* \star} P<0.01$, ${ }^{* * *} P<0.001$. 





Figure 5 RIN2 promotes the recruitment of R-Ras to Rab5-positive early endosomes. Fluorescent confocal microscopy analysis of ECs transfected with GFP-Rab5A (central left panels, green), mCherry-R-Ras 38V (central right panels, red) and HA$m$ RIN2 (A) or HA-mRIN2 RH/VPS9 (B) or HA-mRIN2 RA (C) (left panels, blue). Right panels correspond to merged image. (A) HA-mRIN2, GFP-Rab5A, and mCherry-R-Ras 38V colocalize on early endosomes. (B) HA-mRIN2 RH/VPS9 and mCherryR-Ras 38V colocalize in the perinuclear vesicular compartment, but they are excluded from the peripheral Rab5-labeled early endosomes. (C) HA-mRIN2 RA colocalizes with GFP-Rab5A in peripheral early endosomes, whereas mCherry-R-Ras 38V is excluded from these compartments. Lower panels are magnifications of the corresponding upper panels. (D) Quantification of colocalization signal between: (1) mRIN2 constructs and GFP-Rab5; (2) mRIN2 constructs and mCherry-R-Ras 38V; (3) mCherry-R-Ras 38V and GFP-Rab5 in ECs simultaneously transfected with different $m$ RIN2 constructs (mean \pm SD; $n=25$ cells per experimental condition). Scale bars, $10 \mu \mathrm{m} .{ }^{* * *} P<0.001$.

Rab5 could require both R-Ras and RIN2. To verify this hypothesis, we transduced human ECs first with a retrovirus encoding WT Rab5A and then with lentiviruses carrying shRNAs able to target either R-Ras or RIN2. As expected [62], the overexpression of WT Rab5A strongly promoted EC adhesion to different ECM components (Figure 6B). Moreover, we found that the increase of EC adhesion elicited by Rab5A is fully dependent on the presence of both R-Ras and RIN2 (Figure 6B). Thus, in ECs, RIN2 appears to act as a docking adaptor protein that, on early endosomes, initiates the formation of a synergistic platform hinged on R-Ras and Rab5 GTPases that then sparks off a pro-adhesive signal. To further validate this concept, we retrovirally overexpressed either WT R-Ras, or constitutively active R-Ras $38 \mathrm{~V}$ or RIN2 in human ECs that were then silenced with either a pool of three siRNAs targeting the three human isoforms of Rab5, namely Rab5A, B, and C (sihRab5ABC), or control non-targeting siRNA (siCtl) (Figure 6C). Shortterm adhesion assays showed that knocking down Rab5 proteins significantly impaired both R-Ras- and RIN2elicited EC adhesion (Figure 6D), further demonstrating that these three proteins constitute the backbone of a new signaling pathway that controls endothelial spreading over ECM proteins.

The R-Ras/RIN2/Rab5 signaling module controls GTP loading of Rac1 in ECs

GTP loading of Rac1, a key regulator of adhesion-induced cell spreading [63], can be initiated by both R-Ras [36-38] and Rab5 [31]. These observations, together with our finding that to stimulate EC adhesion to the ECM, RIN2 needs to associate with active Rab5 and R-Ras on early endosomes, suggest that on this vesicular compartment the R-Ras/RIN2/Rab5 complex could activate Rac1 to promote EC adhesion and spreading over the ECM.

In accordance with what was previously reported by Palamidessi et al. [31], fluorescent confocal microscopy analysis revealed that overexpression of GFP-Rab5A, but not GFP alone in ECs (Figure 7A and Supplementary information, Figure S10), elicits the translocation of endogenous Rac1 onto early endosomes (Figure 7B and Supplementary information, Figure S10). In addition, we found that WT HA-tagged $m$ RIN2 localizes together with Rab5 and Rac1 on these vesicles (Figure 7B and Supplementary information, Figure S10). However, we observed that even without Rab5 overexpression, GFP$\mathrm{R}-\mathrm{R}$ as $38 \mathrm{~V}$ was able to promote the relocation of Rac1 onto endosomes per se (Figure 7C and Supplementary information, Figure S10) and this effect was maximized when GFP-R-Ras 38V was co-transfected together with $m$ RIN2 in ECs (Figure 7D and Supplementary information, Figure S10). In addition, we found that a constitutively active Rac1 mutant (Q61L) was able to efficiently rescue the adhesion defects of shhRIN2 ECs to different ECM ligands (Supplementary information, Figure S11A). All together, these data prompted us to directly test whether the R-Ras/RIN2/Rab5 module could be involved in Rac1 activation in ECs. To this aim, we measured Rac1 activity by an affinity assay in which Rac1GTP was pulled down from lysates of adherent ECs incubated with a GST fused to the Cdc42/Rac Interactive Binding (CRIB) region of the Rac1-GTP effector p21activated kinase1 (GST-CRIB) [64]. When compared to cells transduced with control lentiviral shRNA (shCtl), $\operatorname{sh} h$ RIN2 ECs clearly displayed a $50 \%$ reduction of active Rac1-GTP (Figure 8A). Furthermore, as expected $[36,37]$, retrovirus-mediated delivery of constitutively active R-Ras $38 \mathrm{~V}$ in ECs elicited a 1.7-fold activation of Rac1 that however was inhibited by RIN2 silencing (Figure 8A). Hence, in ECs, RIN2 is required for both basal and R-Ras-triggered GTP loading of Rac1. Next, we evaluated the capacity of retrovirus-delivered full-length and deletion constructs of $m$ RIN2 to rescue the defective activation of Rac1 observed in sh $h$ RIN2 ECs (Figure 8B). Compared to WT $m$ RIN2, both $m$ RIN2 $\Delta \mathrm{RH} /$ VPS9 and $m$ RIN2 $\Delta$ RA constructs not only were unable to rescue, but in fact enhanced the defect in GTP loading of Rac1 of adherent sh $h$ RIN2 ECs (Figure 8B). The finding that $m$ RIN $2 \Delta \mathrm{RH} / \mathrm{VPS} 9$ and $m \mathrm{RIN} 2 \Delta \mathrm{RA}$ deletion constructs display dominant-negative effects both in short-term adhesion assays (Figure 4D) and GTP load- 
A

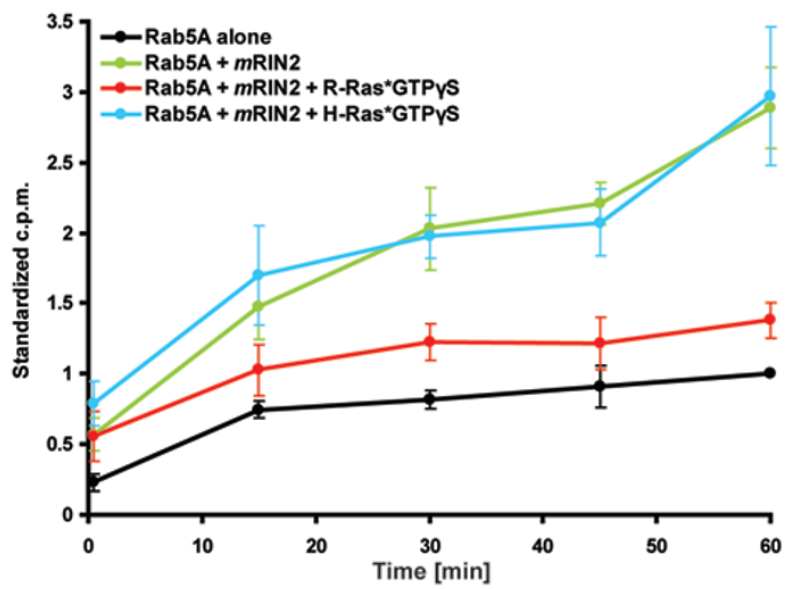

C

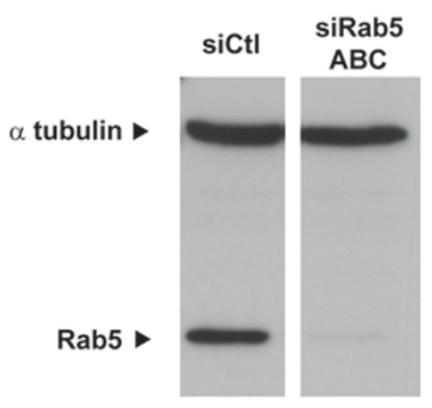

B

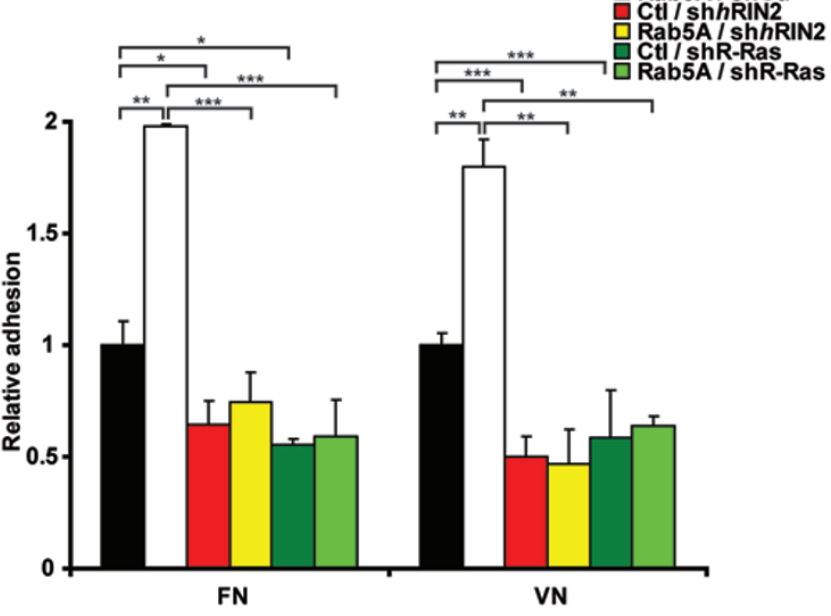

D

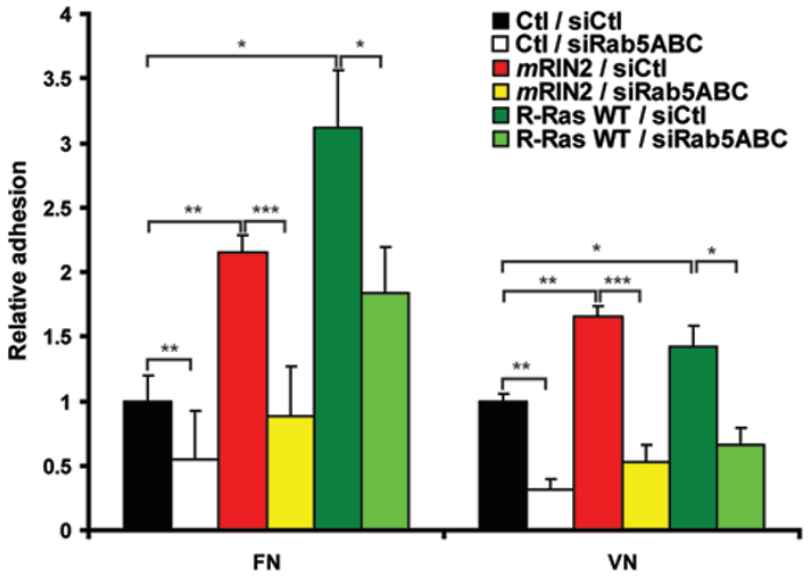

Figure 6 R-Ras, RIN2, and Rab5 constitute a new signaling module that controls the adhesion of ECs to the ECM. (A) The GEF activity of mRIN2 on Rab5A is measured by a nucleotide exchange assay from unlabeled prebound GDP to [ ${ }^{3} \mathrm{H}$ ]-GDP on GST-Rab5A. The rate of $\left.{ }^{3} \mathrm{H}\right]$-GDP association is presented as a correlation of measured counts per minute at each time point for each sample divided by the counts per minute after $60 \mathrm{~min}$ for the control sample Rab5A alone ("standardized c.p.m."). The GEF activity of RIN2 on Rab5A is inhibited by the presence of R-Ras*GTP $\gamma$ S, but not H-Ras*GTP $\gamma$ S. Error bars correspond to the standard error of the mean. (B) Comparative analysis of the modulation of cell adhesion to FN and VN in control (shCtl) or $h$ RIN2 (shhRIN2)- or R-Ras (shR-Ras)-silenced human ECs overexpressing Rab5A or empty PINCO retroviral vector (Ctl). Error bars correspond to standard deviation. A representative of three independent experiments with similar results is shown (mean $\pm \mathrm{SD} ; n=3$ samples per experimental condition). (C) Western blot analysis of Rab5 expression in human ECs silenced for Rab5A, B, C (sihRab5ABC) or transfected with control siRNA (siCtl). $\beta$-tubulin was used as an internal and loading control. (D) Comparative analysis of the modulation of cell adhesion to FN and VN by overexpression of $m \mathrm{RIN} 2$ or R-Ras WT in control (siCtl) or $h$ Rab5ABC (sihRab5ABC)-silenced human ECs. Ctl corresponds to ECs transfected with the empty PINCO retroviral vector. A representative of three independent experiments with similar results is shown (mean \pm SD; $n=3$ samples per experimental condition). ${ }^{*} P<0.05,{ }^{* *} P<0.01,{ }^{* * *} P<0.001$.

ing of Rac1 (Figure 8B) further supports the notion that Rac1 activation constitutes a key molecular mechanism through which the R-Ras/RIN2/Rab5 complex controls $\mathrm{EC}$ adhesion to the ECM.

Finally, we decided to investigate which Rac1 GEFs could mediate the pro-adhesive signaling triggered by the R-Ras/RIN2/Rab5 module. Since both the Rab5- and Ras-binding domains of RIN2 are required to stimulate cell adhesion (Figure 4), Rac1 GTP loading (Figure 8B) and active R-Ras localization on Rab5-positive endo- 
A

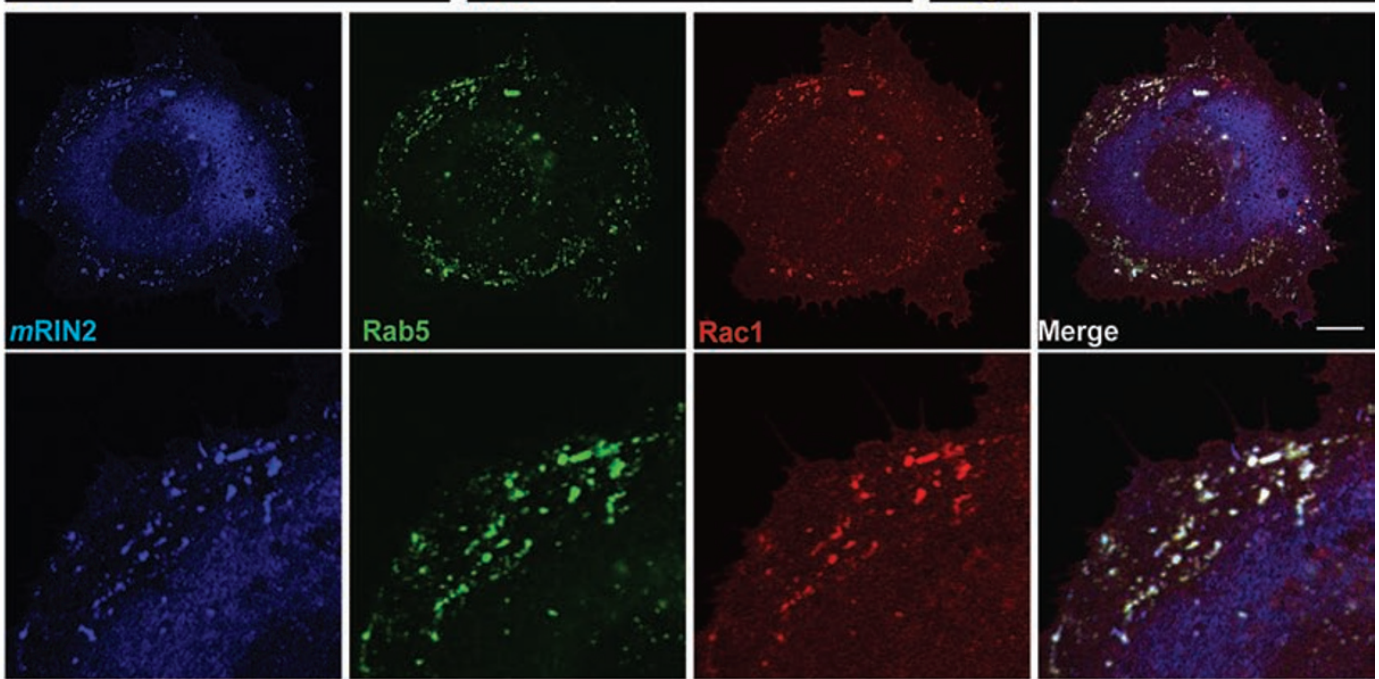

C

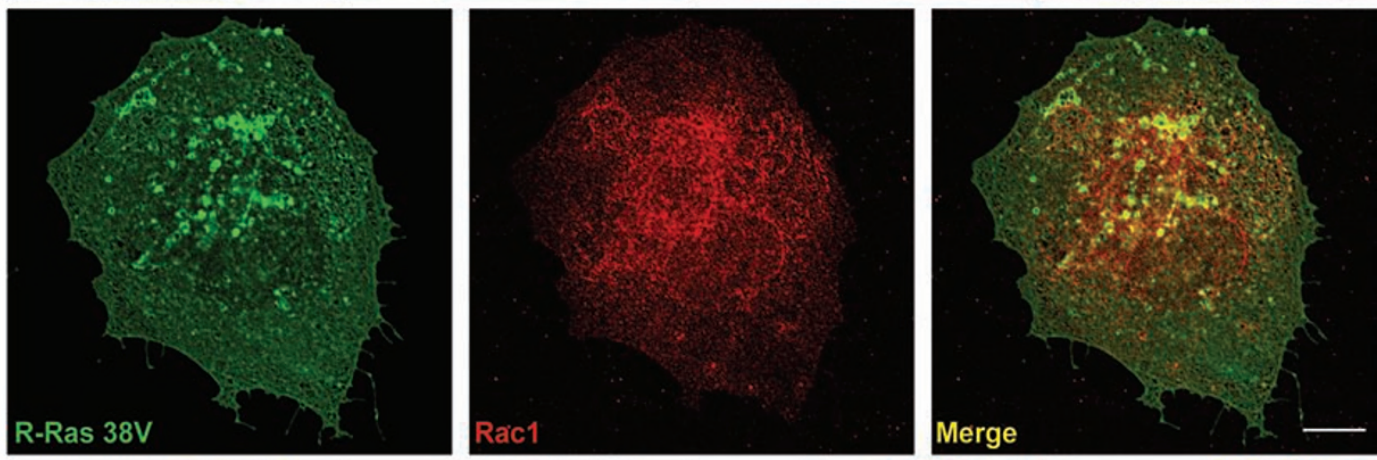

D

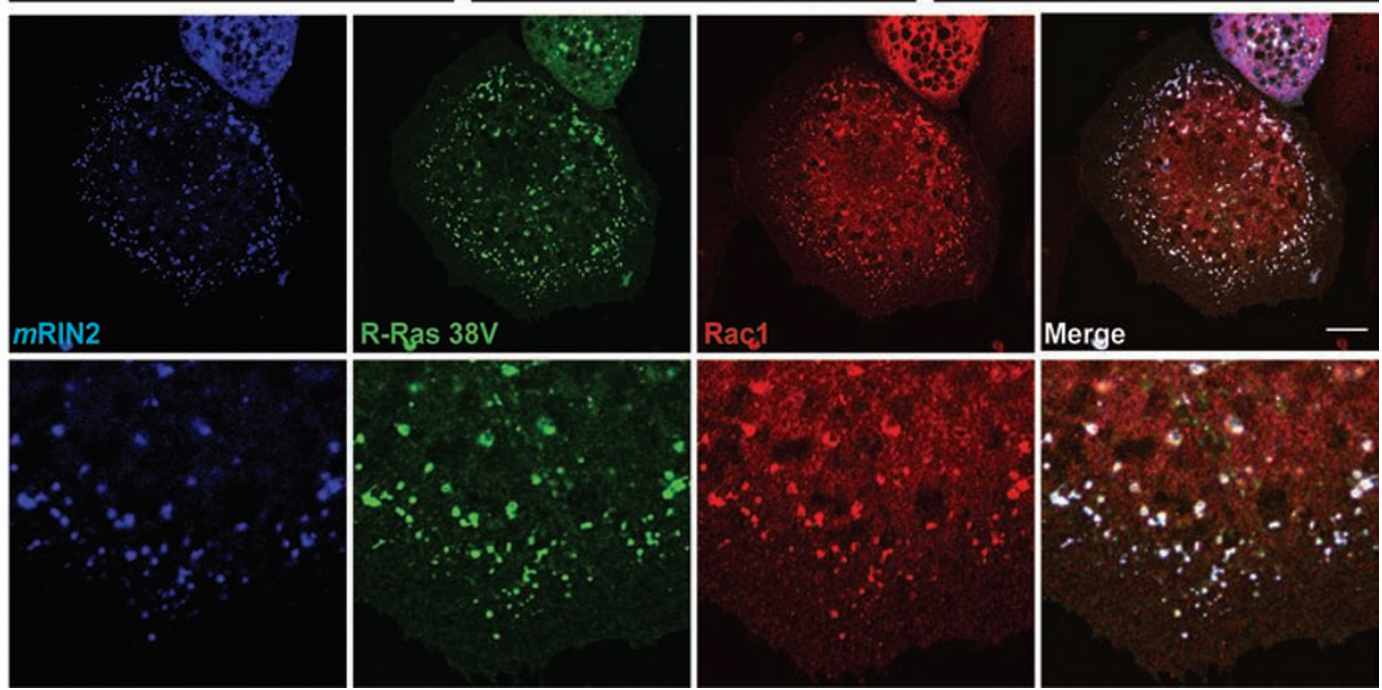


Figure 7 RIN2, R-Ras and Rac1 colocalize on Rab5-positive early endosomes. (A) Fluorescent confocal microscopy analysis of ECs transfected with GFP (left panel, green) and stained for endogenous Rac1 (central panel, red) shows how Rac1 is quite homogeneously distributed throughout the cell. Right panel corresponds to merged image. (B) Fluorescent confocal microscopy analysis of ECs transfected with HA-mRIN2 (left panels, blue) and GFP-Rab5A (central left panels, green) and stained for Rac1 (central right panels, red) shows that HA-mRIN2 and Rac1 colocalize in vesicular compartment marked by GFP-Rab5A. Right panels correspond to merged images. Lower panels are magnifications of the corresponding upper panels. (C) Fluorescent confocal microscopy analysis of ECs transfected with GFP-R-Ras 38V (left panel, green) and stained for endogenous Rac1 (central panel, red) shows a partial colocalization of GFP-R-Ras 38V and Rac1 in bona fide vesicular structures. Right panel corresponds to merged image. (D) Fluorescent confocal microscopy analysis of ECs transfected with HA-mRIN2 (left panels, blue) and GFP-R-Ras 38V (central left panels, green) and stained for Rac1 (central right panels, red) shows the colocalization of HA-mRIN2, GFP-R-Ras 38V and Rac1 in vesicular compartments. Right panels correspond to merged images. Lower panels are magnifications of the corresponding upper panels. Panels are representative of more than 25 cells in three independent experiments. Scale bars, $10 \mu \mathrm{m}$.

somes (Figure 5), the candidate Rac1 GEF(s) should be able to associate spatiotemporally with and get activated by the simultaneous presence of R-Ras and some biochemical feature(s) of the early endosomal environment. Of note, the TIAM1 Rac1 GEF fulfills all these requirements [44]: it can be recruited to Rab5-positive endosomes, where it activates Rac1 [31], and displays a Ras-binding RA domain as well as a pleckstrin homology $(\mathrm{PH})$ domain that selectively interacts with PIns(3) $\mathrm{P}[42,45]$, a phosphoinositide enriched on early endosomal membranes [65]. Moreover, TIAM1 can mediate Ras activation of Rac1 via its RA domain [66]. Hence, we investigated whether the pro-adhesive activity of R-Ras/RIN2/Rab5 signaling module would require the TIAM1 Rac1 GEF. We found that ECs were very sensitive to TIAM1 silencing, most likely due to the key pro-survival role of Rac1 in normal cells [67]. Thus, we transduced human HeLa cells first with a retrovirus encoding the WT version of R-Ras, $m$ RIN2, or Rab5A and then transfected these same cells with a pool of four siRNA duplexes targeting human TIAM1 (siTIAM1) or control non-targeting siRNA (siCtl) (Figure 8C). As observed in human ECs, the overexpression of R-Ras, $m$ RIN2, or Rab5A promoted HeLa adhesion to the ECM (Figure 8D). Importantly, we found that the increase of cell adhesion caused by all these three proteins is dependent on the presence of TIAM1 (Figure 8D). Furthermore, TIAM1 overexpression rescued most of the ECM adhesion defect phenotype that characterizes RIN2-silenced ECs (Supplementary information, Figure S11B). Thus, the Rac1 GEF TIAM1 emerges as a key transducer of the pro-adhesive signaling elicited by the R-Ras/RIN2/Rab5 complex.

\section{Discussion}

In vivo, the small GTPase R-Ras is mainly expressed in vascular endothelial and smooth muscle cells, regu- lates blood vessel remodeling [11], and plays a key role in the control of integrin-mediated cell adhesion to the ECM $[10,18]$. In ECs, SEMA3, either directly or in cooperation with neuropilins, activates PlexinD1 [13, 15] that via its cytoplasmic R-Ras GAP domain, directly switches R-Ras off [68-70] and controls vascular morphogenesis [13-15]. However, the molecular mechanisms by which R-Ras positively influences the ability of ECs to adhere and spread over the ECM are still unknown.

Data from R-Ras overexpression effects in cell types other than ECs have demonstrated that R-Ras does not conformationally activate integrins $[10,21]$ and could instead act by activating Rac1 through undefined signaling pathway(s) $[36,37]$ and/or favoring the RLIP76/ Arf6-mediated targeting of Rac1-GTP to the plasma membrane [38]. Indeed, active Rac1, e.g., by triggering integrin activation [71] and actin polymerization [63], can mediate the R-Ras-elicited and integrin-mediated spreading of ECs on matrix proteins. Moreover, the Rac effector WASH and Arp2/3-mediated actin polymerization have been recently found to control endosomal integrin recycling toward the plasma membrane as well [72]. Likely because of its central role in regulating adhesive and cytoskeletal dynamics, endothelial Rac1 is in fact essential for embryonic vascular morphogenesis [73].

Here, we show that the R-Ras/RIN2/Rab5 signaling complex transiently localizes at NAs and selectively controls the endocytosis of active $\beta 1$ integrins. Importantly, active integrin-mediated ECM binding, likely by triggering GTP loading of R-Ras [58], in turn triggers R-Ras and RIN2 relocation onto Rab5-positive early endosomes, where at steady state the tripartite R-Ras/ RIN2/Rab5 complex preferentially localizes in adherent ECs. In addition, we provide mechanistic evidence of how the RIN2-assisted targeting of R-Ras-GTP onto the Rab5-positive endosomal compartment causes the TIAM1-mediated activation of Rac1, finally promoting EC attachment to the ECM. Even if the R-Ras/RIN2/ 
A

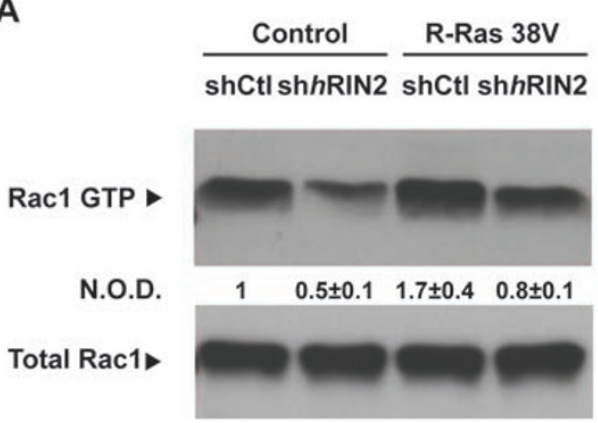

C

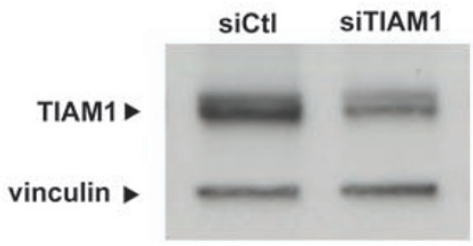

E

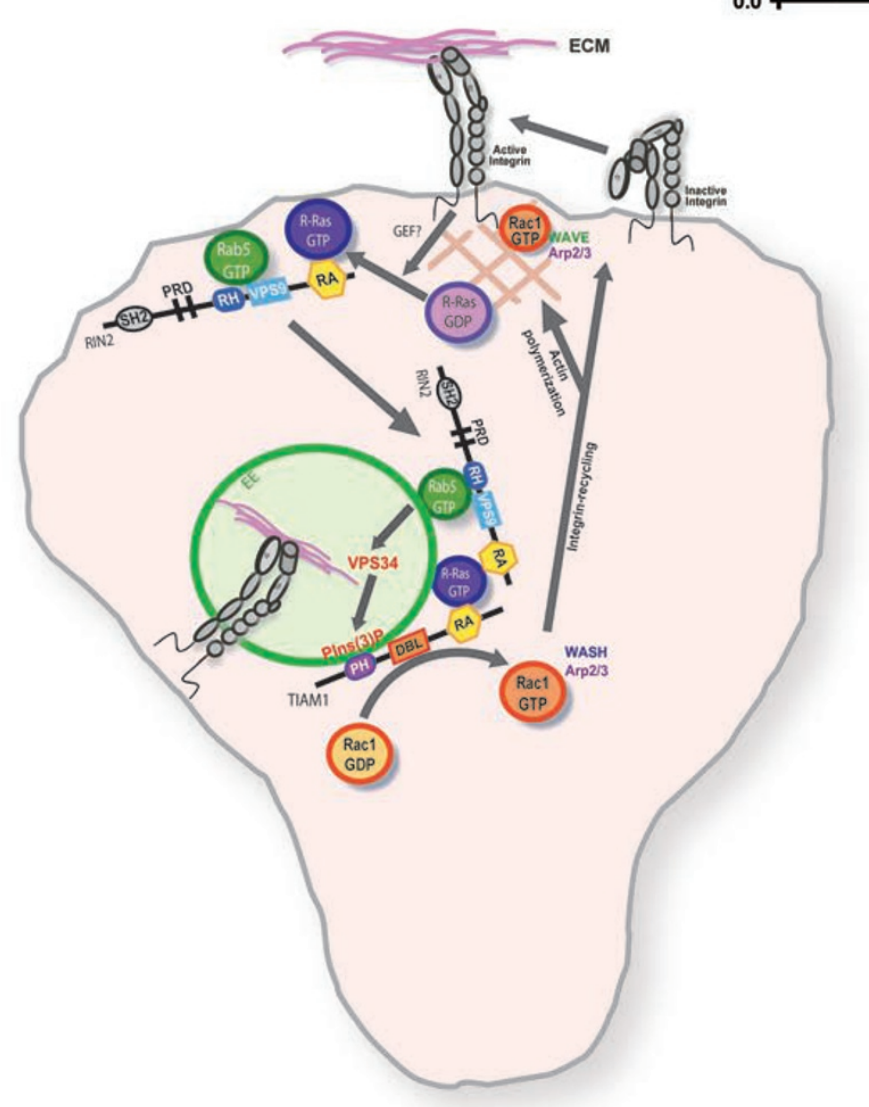

B ShhRIN2 $m$ RIN2 $m$ RIN2 shCtlshhRIN2 mRIN2 $\underset{\text { VRHI }}{m \text { RRA }}$

Rac1 GTP •

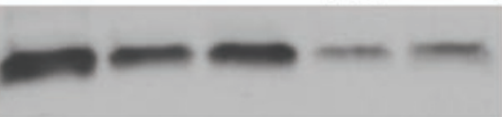

N.O.D.

$\begin{array}{llll}0.5 \pm 0.1 & 0.85 \pm 0.1 & 0.3 \pm 0.1 & 0.2 \pm 0.1\end{array}$

Total Rac1

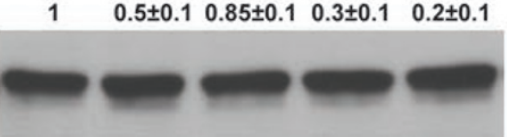

D $\quad$ Ctt/ siCtl

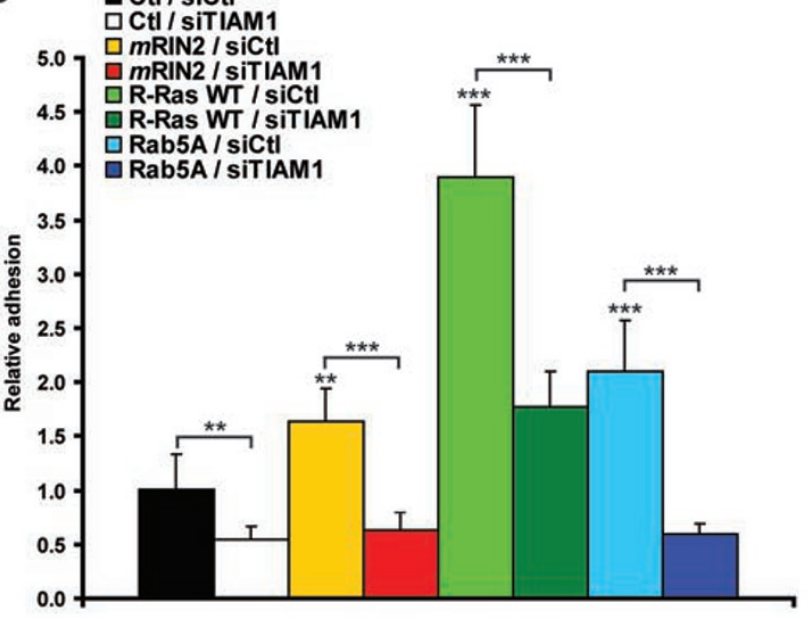


Figure 8 The R-Ras/RIN2/Rab5 module controls Rac1 activation and EC adhesion via TIAM1. (A) Pull-down assay of active Rac1 in ECs overexpressing constitutively active R-Ras $38 \mathrm{~V}$ or the control empty PINCO retroviral vector and silenced for $h$ RIN2 (shhRIN2) or infected with control shRNA (shCtl). Total Rac1, detected in the input fractions, is used to calculate the normalized optical density (N.O.D.) of active Rac1. A representative of three independent pull-down assays with similar results is shown. Bands were quantified and N.O.D.s were calculated relative to control (Values are means \pm SD; $n=3$ separate assays). (B) The ability of $m$ RIN2 or $m R I N 2$ RH/VPS9 or $m R I N 2$ RA to rescue the Rac1 activation defect in control (shCtl) or RIN2-silenced (shhRIN2) ECs was tested by pull-down assay. Total Rac1, detected in the input fractions, is used to calculate the N.O.D. of active Rac1. A representative of three independent pull-down assays with similar results is shown. Bands were quantified and N.O.D.s were calculated relative to control (Values are means \pm SD; $n=3$ separate assays). (C) Western blot analysis of TIAM1 expression in HeLa silenced for TIAM1 (siTIAM1) or transfected with control siRNA (siCtI). Vinculin was used as an internal and loading control. (D) Comparative analysis of the modulation of cell adhesion to FN by overexpression of $m$ RIN2 or R-Ras WT or Rab5A in control (siCtl) or TIAM1 (siTIAM1)-silenced HeLa. Ctl corresponds to HeLa transfected with the empty PINCO retroviral vector. A representative of two independent experiments with similar results is shown (mean $\pm \mathrm{SD} ; n=6-9$ samples per experimental condition). (E) Model of how the R-Ras/RIN2/Rab5 complex controls active integrin endocytosis and signals the TIAM1-dependent activation of Rac1 and EC adhesion to the ECM. At the leading edge of motile ECs, ECM-bound/active integrins trigger GEF-mediated GTP loading of R-Ras. At NAs, active R-Ras interacts with the RA domain of RIN2, inhibits its Rab5 GEF activity, and transforms RIN2 into an adaptor that binds GTP-Rab5 via its RH/VPS9 domain, thus localizing this Rab GTPase at the plasma membrane. Here, the R-Ras/RIN2/Rab5 complex drives the endocytosis of ECM-bound/active integrins and the ensuing translocation of R-Ras and RIN2 proteins to Rab5-positive early endosomes (EE). Also TIAM1, a protein that presents a Ras-binding domain, is recruited to Rab5-positive early endosomes [31]; indeed, TIAM1 possesses a PH domain that binds PIns(3)P, which is highly enriched at the endosomal surface and positively regulates TIAM1 GEF activity [42, 45]. Upon its double binding to PIns(3)P and R-Ras, TIAM1 gets completely activated and acts as a GEF for Rac1. Active Rac1 can promote, in turn, integrin-dependent cell adhesion to the ECM through several mechanisms. Indeed, the Arf6-dependent delivery of Rac1-GTP to the plasma membrane can drive WAVE-Arp2/3-dependent actin polymerization that mediates the formation of new NAs [34]. Furthermore, Rac1-dependent endosomal activation of the WASH-Arp2/3 complex can support the recycling of internalized integrins to the plasma membrane [72], a process that is central to directional cell migration [22]. ${ }^{* *} P<0.01,{ }^{* * *} P<0.001$.

Rab5 complex could trigger GTP loading of Rac1 when transitorily localized at the plasma membrane, the fact that at steady state two major TIAM1 activators, namely active R-Ras (this study and [46]) and PIP3 [74], localize on (early) endosomes strongly points to this vesicular compartment as a preferential site for Rac1 activation downstream of R-Ras.

By silencing-rescue experiments and biochemical analysis, we showed that RIN2 is a key effector protein that physically interacts with and mediates the proadhesive effects of active R-Ras. Notably, while we confirmed that RIN2 [48, 56], similar to RIN3 [61] but different from RIN1 [60] and other Rab5 GEFs [75], preferentially binds Rab5-GTP, we uncovered that active R-Ras but not H-Ras inhibits the Rab5 GEF activity and favors the R-Ras/Rab5 adaptor function of RIN2. The latter finding is of interest in light of the fact that H-Ras and R-Ras have been reported to play opposite and counteracting roles in integrin-mediated adhesion in some cell types [10]. Moreover, based on our finding that the R-Ras/RIN2/Rab5 complex promotes the selective endocytosis of ECM-bound/active $\beta 1$ integrins, it is likely that R-Ras-associated RIN2 docks a GTP-loaded pool of Rab5 at NAs. The amount of Rab5-GTP required to elicit active integrin endocytosis at ECM adhesions could either be derived from the residual Rab5 GEF activity of
RIN2 itself or generated by another Rab5 GEF or, similar to what was described for Rab4 [76], R-Ras-bound RIN2 could extract Rab5-GTP from a cytosolic carrier proteinbuffered pool. Further work is required to clarify this issue.

We found that vesicular localization of R-Ras requires R-Ras activation. In addition, ECM binding to integrins drives R-Ras and RIN2 targeting onto Rab5-positive early endosomes. In particular, GTP-bound R-Ras exploits the docker properties of RIN to promote its own translocation onto early endosomes. We favor the hypothesis that rather at NAs, on this specific vesicular compartment R-Ras-GTP acts as a key element of a signaling platform that finally triggers GTP loading of Rac1 and cell adhesion to the ECM. Indeed, when we reintroduced fulllength and mutant murine RIN2 constructs in human ECs in which endogenous $h$ RIN2 had been simultaneously silenced, we observed that both EC adhesion to ECM proteins and Rac1 GTP loading depend on the RH/VPS9 and RA domains of RIN2 that bind to the active forms of Rab5 and R-Ras, respectively. Importantly, compared to WT $m$ RIN2, overexpression of $m$ RIN2 $\Delta$ RH/VPS9 and $m$ RIN2 $\Delta$ RA failed to rescue the Rac1 activation and cell adhesion defects caused by $h$ RIN 2 silencing, and more importantly, it worsened the phenotypes, indicating that these mutants behave as dominant-negative RIN2 con- 
structs. Furthermore, different from WT $m$ RIN2, both $m$ RIN2 $\Delta$ RH/VPS9 and $m$ RIN2 $\Delta$ RA were almost unable to relocate active R-Ras onto Rab5-positive endosomes. Therefore, the ability of RIN2 to spatially couple R-Ras and Rab5 is responsible for four interconnected events: (1) the endocytosis of ECM-bound/active integrins; (2) the shift of active R-Ras onto early endosomes, where Rac1 also localizes; (3) the R-Ras-dependent GTP loading of Rac1; and (4) the R-Ras-elicited adhesion of ECs to the ECM. In addition, the fact that the overexpression of the constitutively active Rac1 Q61L mutant rescued the adhesion defect caused by RIN2 silencing, further proves the downstream involvement of Rac1 in this pathway. We identified TIAM1 as a Rac1 GEF that mediates the pro-adhesive activity of the R-Ras/RIN2/Rab5 signaling complex. Similar to these three latter proteins, TIAM1 can also localize on Rab5-positive vesicles [31], likely because its $\mathrm{PH}$ domain selectively interacts with the early endosome-enriched phosphatidylinositol PIns(3) $\mathrm{P}[42,45,65]$. Furthermore, the Ras-binding RA domain of TIAM1 allows this GEF to function as an effector that directly mediates the Ras activation of Rac1 [66]. All together, these data support a positive feedback model in which at the leading edge of motile cells ligand-engaged integrins trigger the formation of an R-Ras-GTP/RIN2/ Rab5-GTP complex. This complex drives both the endocytosis of ECM-bound/active integrins themselves and the RIN2-mediated funneling of R-Ras-GTP toward Rab5-containing vesicular compartments to allow the (local) activation of Rac1 by TIAM1, which in turn supports integrin-mediated cell adhesion (Figure 8E).

The need of a RIN2- and Rab5-dependent translocation of R-Ras-GTP onto early endosomes to activate Rac1 and support EC adhesion and spreading on ECM could be due to at least two non-mutually exclusive reasons: polarized endo-exocytic cycling and endosomal signaling (Figure 8E). Indeed, we found that, in spreading ECs, RIN2 colocalizes with active R-Ras both at lamellipodium-associated NAs and on Rab5-containing early endosomes. Of note, in motile cells R-Ras is much more robustly activated at the leading edge than in the cell body [58]. Therefore, it is conceivable that in lamellipodia ECM-bound integrins signal to GTP-loaded R-Ras [58] that thereafter binds to and switches off the Rab5 GEF activity of RIN2. In turn, RIN2, by displaying a much higher affinity for Rab5-GTP than its GDPloaded counterpart, behaves as an adaptor responsible for the translocation of active Rab5 and R-Ras at NAs and onto early endosomes, respectively. While at NAs Rab5GTP elicits the endocytosis of active integrins, on the early endosomal compartment R-Ras-GTP can first trigger the RIN2/TIAM1-dependent activation of Rac1 (this study) and then the RLIP76/Arf6-mediated recycling of Rac1-GTP to lamellipodial protrusions [38] (Figure 8). The fact that Rac1 signals the creation of new lamellipodia-associated NAs [77] would suggest that the R-Ras/ RIN2 signaling module might also be the hub of a positive feedback mechanism aimed at steering cell spreading, by further propagating lamellipodial protrusion and NA formation. Finally, it is now well documented that endosomes, in addition to controlling the recycling or degradation of receptors, have been also evolutionarily exploited as subcellular platforms for the assembly and compartmentalization of signaling complexes in space and time $[57,78]$. The ECM-bound/active integrinelicited and R-Ras-mediated activation of Rac1 on early endosomes could be required to allow the interaction of Rac1-GTP with yet to be identified effector protein(s) not present at the plasma membrane, hence achieving the signaling specificity necessary to control cell adhesion and spreading. A candidate endosomal effector of Rac 1 would for example be represented by the Arp $2 / 3$ activator WASH that controls the recycling of endocytosed $\alpha 5 \beta 1$ integrin to the plasma membrane [72]. From this point of view, endosomes defined by the presence of RIN2 could represent a novel class of signaling endosomes involved in the control of the dynamics of integrin-mediated cell attachment to the ECM.

\section{Materials and Methods}

\section{Antibodies and extracellular matrix proteins}

Mouse monoclonal antibodies (mAbs) anti- $\alpha$-tubulin (B-5-12), anti-R-Ras (2E12) for western blot analysis, and anti-vinculin (hVIN-1) for immunofluorescence analysis were from SigmaAldrich. Mouse mAb anti-RIN2 (ab57377) was from Abcam and used for immunoprecipitation experiments. Mouse mAbs antiRac1 (102), anti-Rab5 (1/Rab5), and anti-LAMP1 (H4A3) were from BD Biosciences. Rabbit anti-RIN2 (HPA034641) and antiRab5 (R4654) antibodies were from Sigma-Aldrich and employed for western blotting. Rat mAb anti-HA High Affinity (3F10), used both for western blot and immunofluorescence analysis, was from Roche. Mouse mAb HA-probe (F-7) and goat polyclonal antiEEA1 (N-19) for immunofluorescence analysis, rabbit polyclonal anti-TIAM1 (C-16), rabbit polyclonal anti- $\beta$-tubulin $(\mathrm{H}-235)$ and mouse monoclonal anti-vinculin (H-10) for western blot analysis were all from Santa Cruz Biotechnology. Rabbit polyclonal anti-GFP (A11122) was from Invitrogen. Rabbit polyclonal antihuman-TGN46 (AHP1586) for immunofluorescence analysis was from $\mathrm{AbD}$ Serotec. In endocytosis assays, total and active $\beta 1$ integrins were detected by mouse mAb JB1B (ab30388) from Abcam and rat mAb 9EG7 (550531) from BD Biosciences, respectively.

Goat anti-rabbit and anti-rat secondary antibodies were from Santa Cruz Biotechnology, while goat anti-mouse secondary antibody was from Jackson ImmunoResearch Laboratories. Alexa Fluor 647 and 555 goat anti-mouse IgG2a, Alexa Fluor 488 and 555 donkey anti-mouse $\operatorname{IgG}(\mathrm{H}+\mathrm{L})$, Alexa Fluor 555 goat anti- 
mouse IgG2b and Alexa Fluor 647 chicken anti-rat IgG secondary antibodies were from Invitrogen.

Human FN was from R\&D Systems. Human plasma VN and Coll I were from Sigma-Aldrich. Rhodamine-labeled bovine FN was from Cytoskeleton Inc.

\section{DNA constructs}

The constructs of pEGFP-R-Ras WT, pEGFP-R-Ras CA (38V) and pEGFP-R-Ras DN (43N) were kindly provided by Johan Peranen (University of Helsinki, Finland). Using standard PCR protocols we transferred these cDNAs in PINCO retrovirus or pGEX4T1 (Amersham Biosciences) vectors. cDNAs of R-Ras 38V and WT were also subcloned in pAcmCherry-N1 vector (derived from the Clontech pAcGFP-N1 vector whose GFP-coding sequence was substituted with the cDNA of the red fluorescent protein mCherry).

pEGFP-Rab7 and Rab5-pCX4-bsr-mEGFP constructs were kindly donated by Cecilia Bucci (University of Salento, Italy) and Michiyuki Matsuda (Kyoto University, Japan), respectively, while Rab5A-GST and Rab5A-GFP/YFP constructs were from Marino Zerial (Max Plank Institute of Molecular Cell Biology and Genetics, Dresden, Germany). Silvio Gutkind (National Institutes of Health, USA) kindly provided the construct of Rac1 Q61L/pcDNA 3.

The HA-tagged $m$ RIN2 construct was generated by standard PCR protocols according to the Taq polymerase manufacturer's instructions (Fynnzymes), using $m$ RIN2/pCMV-Sport6 (Invitrogen) as template and phCMV-2 (Genlantis) as host expression vector. Deletion mutant $m$ RIN2 constructs were obtained with standard molecular biology techniques. In particular, the mutant $m$ RIN2 $\triangle$ RH/VPS9 was lacking in $1363-2214$ base pairs (aa 455-738), while the mutant $m$ RIN2 $\Delta$ RA was devoid of $2251-2508$ base pairs (aa 751-836). Afterwards, these mutants were also subcloned in PINCO retrovirus or pGEX-4T1 vectors.

\section{Gene silencing}

For siRNA-mediated silencing, the day before oligofection, ECs were seeded in 6-well dishes at a concentration of $10 \times 10^{4}$ cells/well. Oligofection of siRNA duplexes was performed according to manufacturer's protocols. Briefly, human ECs or HeLa were transfected twice (at 0 and $24 \mathrm{~h}$ ) with 200 pmol of siCONTROL non-targeting siRNA \#1 (as control) or siGENOME SMART pools (Dharmacon) for human R-Ras (M-010352-02) or RIN2 (M008173-01) or TIAM1 (M-003932-02). 24 h (R-Ras and RIN2) or $48 \mathrm{~h}$ (TIAM1) after the second oligofection ECs or HeLa were lysed or tested in functional assays. To silence Rab5A, B and C, human ECs were transfected with 375 pmol of Stealth RNAinegative control duplexes (medium GC duplex) or 200 pmol of each of HSS184174 (against human Rab5A), HSS108980 (against human Rab5B) and HSS143567 (against human Rab5C), provided by Invitrogen. $48 \mathrm{~h}$ after the oligofection, ECs were lysed or tested in functional assays.

Lentiviral vectors carrying shRNA sequences against R-Ras (TRCN0000047885) or RIN2 (TRCN0000062645) were from the RNAi Consortium library (Sigma-Aldrich). Lentiviruses were produced as described in the TRC shRNA guidelines [48].

\section{Adhesion assay}

6000 ECs were resuspended in $0.1 \mathrm{ml}$ of EBM-2 medium (Lonza) and plated on 96-well microtitre plates (Costar) that were previously coated with ECM proteins $(0.3 \mu \mathrm{g} / \mathrm{ml} \mathrm{FN}$ or $1 \mu \mathrm{g} / \mathrm{ml}$
$\mathrm{VN}$ or $0.5 \mu \mathrm{g} / \mathrm{ml}$ Coll I) and then saturated with $3 \%$ bovine serum albumin (BSA). Cells were left to adhere for $20 \mathrm{~min}$ at $37{ }^{\circ} \mathrm{C}, 5 \%$ $\mathrm{CO}_{2}$ in a humidified atmosphere. $70000 \mathrm{HeLa}$ were resuspended in $0.3 \mathrm{ml}$ of Dulbecco's Modified Eagle's Medium (Sigma) and plated on glass coverslips previously coated with $1 \mu \mathrm{g} / \mathrm{ml}$ of FN and saturated with 3\% BSA. Cells were left to adhere for $90 \mathrm{~min}$ at $37^{\circ} \mathrm{C}, 5 \% \mathrm{CO}_{2}$ in a humidified atmosphere.

Cells were fixed in $8 \%$ glutaraldehyde, and then stained with $0.1 \%$ crystal violet and $20 \%$ methanol. Cells were photographed with a QIcam FAST1394 digital color camera (QImaging) and counted by Image-ProPlus 6.2 software (Media Cybernetics). Results were expressed as relative adhesion, i.e., number of control cells adhered to ECM ligands was assigned the value of 1-fold $(100 \%)$ and relative adhesion was calculated.

\section{FACS analysis of FN binding to ECs}

ECs were detached, counted (150 000 cells/sample), washed once with $1 \mathrm{ml}$ of Tyrode's buffer (10 mM HEPES-NaOH, pH 7.5, $137 \mathrm{mM} \mathrm{NaCl}, 2.68 \mathrm{mM} \mathrm{KCl}, 0.42 \mathrm{mM} \mathrm{NaH}_{2} \mathrm{PO}_{4}, 1.7 \mathrm{mM} \mathrm{MgCl}_{2}$, $11.9 \mathrm{mM} \mathrm{NaHCO}_{3}$, and $5 \mathrm{mM}$ glucose) [79] at $4{ }^{\circ} \mathrm{C}$, resuspended in Tyrode's buffer containing or not $15 \mu \mathrm{g} / \mathrm{ml}$ of rhodamine-FN, and incubated for $30 \mathrm{~min}$ at room temperature (RT). Cells were then washed twice with Tyrode's buffer at $4{ }^{\circ} \mathrm{C}$ and finally analyzed by FACS with a CyAN ADP analyzer (Beckman Coulter).

\section{In vitro angiogenesis assay}

16000 ECs were resuspended in $350 \mu \mathrm{l}$ of $10 \%$ FBS EBM-2, plated in each well of a 48 -well plate previously coated with 150 $\mu \mathrm{l} / \mathrm{well}$ of $8 \mathrm{mg} / \mathrm{ml}$ growth factor-reduced Matrigel (BD Biosciences), and incubated at $37{ }^{\circ} \mathrm{C}, 5 \% \mathrm{CO}_{2}$ in a humidified atmosphere for $5 \mathrm{~h}$ to allow vascular network formation. Samples were then observed by a Leica DMI 3000 B inverted microscope (Leica Microsystems). Images were acquired with a CoolSNAP HQ camera (Photometrics) controlled by Image Pro Plus software (Media Cybernetics) and the number of branching points per $\mathrm{mm}^{2}$ were analyzed and counted with ImageJ software (Wayne Rasband, National Institutes of Health, USA) as previously described [80].

\section{Wound healing assay}

200000 ECs were plated in each well of a 24-well plate previously coated with $3 \mu \mathrm{g} / \mathrm{ml}$ human plasma $\mathrm{FN}$ and incubated overnight at $37{ }^{\circ} \mathrm{C}, 5 \% \mathrm{CO}_{2}$ in a humidified atmosphere. The day after, each well was scratched with a $200 \mu$ Gilson pipette tip in the middle of the monolayer. Cells were then incubated at $37^{\circ} \mathrm{C}$, $5 \% \mathrm{CO}_{2}$ in a humidified atmosphere in an AF6000 LX workstation (Leica Microsystems) and imaged by time-lapse phase-contrast videomicroscopy. Frames were taken every 15 min for $12 \mathrm{~h}$. Displacement of the endothelial sheet leading edge over time was quantified by LAS AF Leica Application Suite software (Leica Microsystems).

\section{Confocal scanning microscopy}

Cells were plated on glass coverslips coated with $5 \mu \mathrm{g} / \mathrm{ml} \mathrm{FN}$ and allowed to adhere for $3 \mathrm{~h}$. Cells were washed in phosphatebuffered saline (PBS), fixed in 4\% para-formaldehyde (PFA), permeabilized in $0.01 \%$ saponin for $5 \mathrm{~min}$ on ice, incubated with different primary antibodies for $1 \mathrm{~h}$, and revealed by appropriate Alexa-Fluor-tagged secondary antibody (Molecular Probes by Life Technologies). HA- $m$ RIN2, HA- $m$ RIN2 $\Delta$ RH/VPS9 and HA- 
$m$ RIN2 $\triangle$ RA were stained with a mouse anti-HA antibody and the Alexa-405-tagged secondary antibody. Rac1 was detected by an anti-Rac1 antibody and the Alexa-555-tagged secondary antibody. Cells were analyzed by using a Leica TCS SP2 AOBS confocal laser-scanning microscope (Leica Microsystems). Immunofluorescence analysis was performed as previously described [49, 50]. Colocalization analysis was performed with the Imaris 6.3 (Bitplane, AG) software colocalization module. We also quantified fluorescence intensity profiles along linear regions of interest (ROI) in multiple channels by the Leica Confocal Software Quantification Tool (Leica Microsystems). Image acquisition was performed by adopting a laser power, gain, and offset settings that allowed maintaining pixel intensities (gray scale) within the 0-255 range and hence avoided saturation.

In some immunofluorescence experiments, ECs were plated on FN $(5 \mu \mathrm{g} / \mathrm{ml})$-coated coverslips in a 24-well plate and the day after they were treated with $20 \mu \mathrm{M}$ Blebbistatin (Sigma-Aldrich) diluted in M199 complete medium for $1 \mathrm{~h}$ at $37{ }^{\circ} \mathrm{C}, 5 \% \mathrm{CO}_{2}$ in a humidified atmosphere. Afterwards, cells were gently washed and fixed in $4 \%$ PFA.

To stain suspended ECs, cells were detached, counted, centrifuged at $800 \times g$ RT, resuspended in poor M199 medium, and kept in suspension on ice for $30 \mathrm{~min}$ to allow a complete switch-off of ECM-dependent integrin signaling. Cells were then re-pelleted at $800 \times g$ RT, resuspended in $1 \mathrm{ml}$ of prewarmed poor M199 medium containing or not $30 \mu \mathrm{g} / \mathrm{ml}$ of human plasma $\mathrm{FN}$, and incubated for $5 \mathrm{~min}$ at $37{ }^{\circ} \mathrm{C}, 5 \% \mathrm{CO}_{2}$ in a humidified atmosphere. ECs were then washed once with ice-cold PBS, centrifuged at $800 \times g$ RT, put back on ice, fixed in 4\% PFA, and then permeabilized for 5 min with $0.01 \%$ saponin in PBS on ice. Cells were then washed once in PBS, stained, and finally centrifuged and resuspended in mounting medium, $10 \mu 1$ of which were then plated on a slide before being covered with a coverslip. Confocal stack images were digitally post-processed with background subtraction and blind deconvolution algorithms of Imaris 6.3 software. Colocalization analysis was then performed with the Imaris 6.3 software colocalization module.

\section{Integrin internalization assay}

Integrin traffic assays were performed as previously described by Roberts et al. [81] with minor modifications. Cells were transferred to ice, washed twice in cold PBS, and surface-labeled at 4 ${ }^{\circ} \mathrm{C}$ with $0.2 \mathrm{mg} / \mathrm{ml}$ sulfo-NHS-SS-biotin (Pierce) in PBS for 30 min. Labeled cells were washed in cold PBS and transferred to prewarmed M199 containing 10\% FBS and $0.1 \mathrm{mM}$ Primaquine at $37^{\circ} \mathrm{C}$. At the indicated times $(5,10$, and $15 \mathrm{~min})$, the medium was removed, and dishes were rapidly transferred to ice and washed twice with ice-cold PBS. Biotin was stripped from proteins remaining at the cell surface by incubation with a solution containing $20 \mathrm{mM}$ sodium 2-mercaptoethanesulfonate (MesNa) in $50 \mathrm{mM}$ Tris- $\mathrm{HCl}(\mathrm{pH} \mathrm{8.6}), 100 \mathrm{mM} \mathrm{NaCl}$ for $1 \mathrm{~h}$ at $4{ }^{\circ} \mathrm{C}$. MesNa was quenched by the addition of $20 \mathrm{mM}$ iodoacetamide (IAA) for $10 \mathrm{~min}$, and after other two further washes in PBS, the cells were lysed in $25 \mathrm{mM}$ Tris-HCl, $\mathrm{pH} 7.4,100 \mathrm{mM} \mathrm{NaCl}, 2 \mathrm{mM} \mathrm{MgCl}$, $1 \mathrm{mM} \mathrm{Na} \mathrm{VO}_{4}, 0.5 \mathrm{mM}$ (ethylene glycol tetraacetic acid) EGTA, $1 \%$ Triton X-100, 5\% glycerol, protease inhibitor cocktail (SigmaAldrich, $50 \mathrm{mg} / \mathrm{ml}$ pepstatin, $50 \mathrm{mg} / \mathrm{ml}$ leupeptin, and $10 \mathrm{mg} / \mathrm{ml}$ aprotinin), and $1 \mathrm{mM}$ phenylmethanesulfonylfluoride (PMSF). Lysates were cleared by centrifugation at $12000 \times \mathrm{g}$ for $20 \mathrm{~min}$.
Supernatants were corrected to equivalent protein concentrations and levels of biotinylated integrin were determined by capture ELISA assay.

\section{Capture ELISA assay}

Corning 96 Well Clear Polystyrene High Bind Stripwell Microplate (product \#2592) were coated overnight with $5 \mu \mathrm{g} / \mathrm{ml}$ appropriate anti-integrin antibodies in $0.05 \mathrm{M} \mathrm{Na}_{2} \mathrm{CO}_{3}$ (pH 9.6) at $4{ }^{\circ} \mathrm{C}$ and were blocked in PBS containing $0.05 \%$ Tween-20 (PBS-T) with $5 \%$ BSA for $1 \mathrm{~h}$ at RT. Integrins were captured by overnight incubation of $50 \mu \mathrm{l}$ cell lysate at $4{ }^{\circ} \mathrm{C}$. Unbound material was removed by extensive washing with PBS-T, and wells were incubated with streptavidin-conjugated horseradish peroxidase (Amersham) in PBS-T containing $1 \%$ BSA for $1 \mathrm{~h}$ at $4{ }^{\circ} \mathrm{C}$. Following further washing, biotinylated integrins were detected by a chromogenic reaction with ortho-phenylenediamine.

\section{Statistical analysis}

For statistical evaluation, results were analyzed by a two-tailed heteroscedastic Student's $t$-test.

Further information can be found in Supplementary information, Data S1.

\section{Acknowledgments}

We acknowledge Johan Peranen, Cecilia Bucci, Michiyuki Matsuda, Marino Zerial, and Silvio Gutkind for providing reagents. We are grateful to Giorgio Scita and Andrea Palamidessi for suggestions and insightful discussions. We thank Elena Astanina and Giorgio Seano for help in analysis and quantification of confocal images. This work was supported by grants from Telethon Italy (GGP04127 and GGP09175 to GS); Compagnia di San Paolo - Neuroscience Program Multicentre Projects (to GS); Associazione Augusto per la Vita (to GS); Fondazione Guido Berlucchi (to GS); Associazione Italiana per la Ricerca sul Cancro (IG \# 9211 to GS, IG \#10133 to FB, and START UP PROGRAM to LL); Fondazione Piemontese per la Ricerca sul Cancro - ONLUS - Intramural Grant 2010 (to GS and LL); Ministero della Salute - Programma Ricerca Oncologica 2006 and Ricerca Finalizzata 2006 (to GS and FB); Ricerca Finalizzata 2007 (to LL); Regione Piemonte - Ricerca Sanitaria Finalizzata 2006, 2008, and 2009; Ricerca Scientifica Applicata 2004: grants D10 and A150; Ricerca industriale e sviluppo precompetitivo 2006: grants PRESTO and SPLASERBA (to GS, LL and FB); Piattaforme Tecnologiche per le Biotecnologie: grant DRUIDI; Ricerca Tecnologie Convergenti: grant PHOENICS; Ricerca Industriale 2009: grant BANP (to FB); Ministero dell'Università e della Ricerca - Fondo per gli Investimenti della Ricerca di Base: grant NEWTON-RBAP11BYNP (to FB); University of Torino-Progetti di Ateneo 2011: grant Rethe ORTO11RKTW (to FB); and Academy of Finland and ERC Starting grant (to JI). SV is supported by EMBO LTF and Alexander Von Humbolt Foundation.

\section{References}

1 Fraisl P, Mazzone M, Schmidt T, Carmeliet P. Regulation of angiogenesis by oxygen and metabolism. Dev Cell 2009; 
16:167-179.

2 Jones EA, le Noble F, Eichmann A. What determines blood vessel structure? Genetic prespecification vs. hemodynamics. Physiology (Bethesda) 2006; 21:388-395.

3 Adams RH, Alitalo K. Molecular regulation of angiogenesis and lymphangiogenesis. Nat Rev Mol Cell Biol 2007; 8:464478.

4 Jain RK. Lessons from multidisciplinary translational trials on anti-angiogenic therapy of cancer. Nat Rev Cancer 2008; 8:309-316.

5 Jain RK. Normalization of tumor vasculature: an emerging concept in antiangiogenic therapy. Science 2005; 307:58-62.

6 Serini G, Valdembri D, Bussolino F. Integrins and angiogenesis: a sticky business. Exp Cell Res 2006; 312:651-658.

7 Hynes RO. Cell-matrix adhesion in vascular development. $J$ Thromb Haemost 2007; 5 Suppl 1:32-40.

8 van der Flier A, Badu-Nkansah K, Whittaker CA, et al. Endothelial alpha5 and alphav integrins cooperate in remodeling of the vasculature during development. Development 2010; 137:2439-2449.

9 Colicelli J. Human RAS superfamily proteins and related GTPases. Sci STKE 2004; 2004:RE13.

10 Kinbara K, Goldfinger LE, Hansen M, Chou FL, Ginsberg MH. Ras GTPases: integrins' friends or foes? Nat Rev Mol Cell Biol 2003; 4:767-776.

11 Komatsu M, Ruoslahti E. R-Ras is a global regulator of vascular regeneration that suppresses intimal hyperplasia and tumor angiogenesis. Nat Med 2005; 11:1346-1350.

12 Erdmann J, Grosshennig A, Braund PS, et al. New susceptibility locus for coronary artery disease on chromosome $3 \mathrm{q} 22.3$. Nat Genet 2009; 41:280-282.

13 Gitler AD, Lu MM, Epstein JA. PlexinD1 and semaphorin signaling are required in endothelial cells for cardiovascular development. Dev Cell 2004; 7:107-116.

14 Torres-Vazquez J, Gitler AD, Fraser SD, et al. Semaphorinplexin signaling guides patterning of the developing vasculature. Dev Cell 2004; 7:117-123.

15 Zhang Y, Sing MK, Degenhardt KR, et al. Tie2Cre-mediated inactivation of plexinD1 results in congenital heart, vascular and skeletal defects. Dev Biol 2009; 325:82-93.

16 Uesugi K, Oinuma I, Katoh H, Negishi M. Different requirement for Rnd GTPases of R-Ras GAP activity of Plexin-C1 and Plexin-D1. J Biol Chem 2009; 284:6743-6751.

17 Roodink I, Raats J, van der Zwaag B, et al. Plexin D1 expression is induced on tumor vasculature and tumor cells: a novel target for diagnosis and therapy? Cancer Res 2005; 65:8317-8323.

18 Zhang Z, Vuori K, Wang H, Reed JC, Ruoslahti E. Integrin activation by R-ras. Cell 1996; 85:61-69.

19 Moser M, Legate KR, Zent R, Fassler R. The tail of integrins, talin, and kindlins. Science 2009; 324:895-899.

20 Shattil SJ, Kim C, Ginsberg MH. The final steps of integrin activation: the end game. Nat Rev Mol Cell Biol 2010; 11:288300 .

21 Sethi T, Ginsberg MH, Downward J, Hughes PE. The small GTP-binding protein R-Ras can influence integrin activation by antagonizing a Ras/Raf-initiated integrin suppression pathway. Mol Biol Cell 1999; 10:1799-1809.

22 Caswell PT, Vadrevu S, Norman JC. Integrins: masters and slaves of endocytic transport. Nat Rev Mol Cell Biol 2009; 10:843-853.

23 Ivaska J, Heino J. Cooperation between integrins and growth factor receptors in signaling and endocytosis. Annu Rev Cell Dev Biol 2011; 27:291-320.

24 Margadant C, Monsuur HN, Norman JC, Sonnenberg A. Mechanisms of integrin activation and trafficking. Curr Opin Cell Biol 2011; 23:607-614.

25 Valdembri D, Caswell PT, Anderson KI, et al. Neuropilin-1/ GIPC1 signaling regulates alpha5beta 1 integrin traffic and function in endothelial cells. PLoS Biol 2009; 7:e25.

26 Dozynkiewicz MA, Jamieson NB, Macpherson I, et al. Rab25 and CLIC3 collaborate to promote integrin recycling from late endosomes/lysosomes and drive cancer progression. Dev Cell 2012; 22:131-145.

27 Arjonen A, Alanko J, Veltel S, Ivaska J. Distinct recycling of active and inactive $\beta 1$ integrins. Traffic 5 January 2012. doi:10.1111/j.1600-0854.2012.01327.x

28 Valdembri D, Sandri C, Santambrogio M, Serini G. Regulation of integrins by conformation and traffic: it takes two to tango. Mol Biosyst 2011; 7:2539-2546.

29 Ribeiro I, Yuan L, Tanentzapf G, Dowling JJ, Kiger A. Phosphoinositide regulation of integrin trafficking required for muscle attachment and maintenance. PLoS Genet 2011; 7:e1001295.

30 Sandilands E, Frame MC. Endosomal trafficking of Src tyrosine kinase. Trends Cell Biol 2008; 18:322-329.

31 Palamidessi A, Frittoli E, Garre M, et al. Endocytic trafficking of Rac is required for the spatial restriction of signaling in cell migration. Cell 2008; 134:135-147.

32 Sandilands E, Cans C, Fincham VJ, et al. RhoB and actin polymerization coordinate Src activation with endosomemediated delivery to the membrane. Dev Cell 2004; 7:855-869.

33 Vicente-Manzanares M, Choi CK, Horwitz AR. Integrins in cell migration - the actin connection. J Cell Sci 2009; 122:199206.

34 Parsons JT, Horwitz AR, Schwartz MA. Cell adhesion: integrating cytoskeletal dynamics and cellular tension. Nat Rev Mol Cell Biol 2011; 11:633-643.

35 Choi CK, Vicente-Manzanares M, Zareno J, Whitmore LA, Mogilner A, Horwitz AR. Actin and alpha-actinin orchestrate the assembly and maturation of nascent adhesions in a myosin II motor-independent manner. Nat Cell Biol 2008; 10:10391050 .

36 Holly SP, Larson MK, Parise LV. The unique N-terminus of R-ras is required for Rac activation and precise regulation of cell migration. Mol Biol Cell 2005; 16:2458-2469.

37 Osada M, Tolkacheva T, Li W, et al. Differential roles of Akt, Rac, and Ral in R-Ras-mediated cellular transformation, adhesion, and survival. Mol Cell Biol 1999; 19:6333-6344.

38 Goldfinger LE, Ptak C, Jeffery ED, Shabanowitz J, Hunt DF, Ginsberg MH. RLIP76 (RalBP1) is an R-Ras effector that mediates adhesion-dependent Rac activation and cell migration. $J$ Cell Biol 2006; 174:877-888.

39 Insall RH, Machesky LM. Actin dynamics at the leading edge: from simple machinery to complex networks. Dev Cell 2009; 17:310-322

40 Bucci C, Parton RG, Mather IH, et al. The small GTPase rab5 functions as a regulatory factor in the early endocytic pathway. 
Cell 1992; 70:715-728.

41 Lanzetti L, Palamidessi A, Areces L, Scita G, Di Fiore PP. Rab5 is a signalling GTPase involved in actin remodelling by receptor tyrosine kinases. Nature 2004; 429:309-314.

42 Baumeister MA, Martinu L, Rossman KL, Sondek J, Lemmon MA, Chou MM. Loss of phosphatidylinositol 3-phosphate binding by the C-terminal Tiam-1 pleckstrin homology domain prevents in vivo Rac1 activation without affecting membrane targeting. J Biol Chem 2003; 278:11457-11464.

43 Crompton AM, Foley LH, Wood A, et al. Regulation of Tiam1 nucleotide exchange activity by pleckstrin domain binding ligands. J Biol Chem 2000; 275:25751-25759.

44 Mertens AE, Roovers RC, Collard JG. Regulation of Tiam1Rac signalling. FEBS Lett 2003; 546:11-16.

45 Snyder JT, Rossman KL, Baumeister MA, et al. Quantitative analysis of the effect of phosphoinositide interactions on the function of Dbl family proteins. J Biol Chem 2001; 276:4586845875.

46 Takaya A, Kamio T, Masuda M, et al. R-Ras regulates exocytosis by Rg12/Rlf-mediated activation of RalA on endosomes. Mol Biol Cell 2007; 18:1850-1860.

47 Xu J, Shi S, Matsumoto N, Noda M, Kitayama H. Identification of $\mathrm{Rgl} 3$ as a potential binding partner for Rapfamily small G-proteins and profilin II. Cell Signal 2007; 19:1575-1582.

48 Saito K, Kajiho H, Araki Y, et al. Purification and analysis of RIN family-novel Rab5 GEFs. Methods Enzymol 2005; 403:276-283.

49 Wang Y, Colicelli J. RAS interaction with effector target RIN1. Methods Enzymol 2001; 332:139-151.

50 Astrof S, Hynes RO. Fibronectins in vascular morphogenesis. Angiogenesis 2009; 12:165-175.

51 Rodriguez LG, Wu X, Guan JL. Wound-healing assay. Methods Mol Biol 2005; 294:23-29.

52 Serini G, Ambrosi D, Giraudo E, Gamba A, Preziosi L, Bussolino F. Modeling the early stages of vascular network assembly. EMBO J 2003; 22:1771-1779.

53 Pasapera AM, Schneider IC, Rericha E, Schlaepfer DD, Waterman CM. Myosin II activity regulates vinculin recruitment to focal adhesions through FAK-mediated paxillin phosphorylation. J Cell Biol 2010; 188:877-890.

54 Askari JA, Tynan CJ, Webb SE, Martin-Fernandez ML, Ballestrem C, Humphries MJ. Focal adhesions are sites of integrin extension. J Cell Biol 2010; 188:891-903.

55 Conklin MW, Ada-Nguema A, Parsons M, Riching KM, Keely PJ. R-Ras regulates beta1-integrin trafficking via effects on membrane ruffling and endocytosis. BMC Cell Biol 2010; 11:14.

56 Saito K, Murai J, Kajiho H, Kontani K, Kurosu H, Katada T. A novel binding protein composed of homophilic tetramer exhibits unique properties for the small GTPase Rab5. J Biol Chem 2002; 277:3412-3418.

57 Scita G, Di Fiore PP. The endocytic matrix. Nature 2010; 463:464-473.

58 Wozniak MA, Kwong L, Chodniewicz D, Klemke RL, Keely PJ. R-Ras controls membrane protrusion and cell migration through the spatial regulation of Rac and Rho. Mol Biol Cell 2005; 16:84-96.

59 Hansen M, Prior IA, Hughes PE, et al. C-terminal sequences in R-Ras are involved in integrin regulation and in plasma membrane microdomain distribution. Biochem Biophys Res Commun 2003; 311:829-838.

60 Tall GG, Barbieri MA, Stahl PD, Horazdovsky BF. Ras-activated endocytosis is mediated by the Rab5 guanine nucleotide exchange activity of RIN1. Dev Cell 2001; 1:73-82.

61 Kajiho H, Saito K, Tsujita K, et al. RIN3: a novel Rab5 GEF interacting with amphiphysin II involved in the early endocytic pathway. J Cell Sci 2003; 116:4159-4168.

62 Pellinen T, Arjonen A, Vuoriluoto K, Kallio K, Fransen JA, Ivaska J. Small GTPase Rab21 regulates cell adhesion and controls endosomal traffic of beta1-integrins. J Cell Biol 2006; 173:767-780.

63 Heasman SJ, Ridley AJ. Mammalian Rho GTPases: new insights into their functions from in vivo studies. Nat Rev Mol Cell Biol 2008; 9:690-701.

64 Waterman-Storer CM, Worthylake RA, Liu BP, Burridge K, Salmon ED. Microtubule growth activates Rac1 to promote lamellipodial protrusion in fibroblasts. Nat Cell Biol 1999; 1:45-50.

65 Nicot AS, Laporte J. Endosomal phosphoinositides and human diseases. Traffic 2008; 9:1240-1249.

66 Lambert JM, Lambert QT, Reuther GW, et al. Tiam1 mediates Ras activation of Rac by a PI(3)K-independent mechanism. Nat Cell Biol 2002; 4:621-625.

67 Mettouchi A, Klein S, Guo W, et al. Integrin-specific activation of Rac controls progression through the G(1) phase of the cell cycle. Mol Cell 2001; 8:115-127.

68 Kruger RP, Aurandt J, Guan KL. Semaphorins command cells to move. Nat Rev Mol Cell Biol 2005; 6:789-800.

69 Neufeld G, Kessler O. The semaphorins: versatile regulators of tumour progression and tumour angiogenesis. Nat Rev Cancer 2008; 8:632-645.

70 Sakurai A, Gavard J, Annas-Linhares Y, et al. Semaphorin 3E initiates antiangiogenic signaling through Plexin D1 by regulating Arf6 and R-Ras. Mol Cell Biol 2010; 30:3086-3098.

71 Bolomini-Vittori M, Montresor A, Giagulli C, et al. Regulation of conformer-specific activation of the integrin LFA-1 by a chemokine-triggered Rho signaling module. Nat Immunol 2009; 10:185-194.

72 Zech T, Calaminus SD, Caswell $\mathrm{P}$, et al. The Arp2/3 activator WASH regulates $\alpha 5 \beta 1$-integrin-mediated invasive migration. $J$ Cell Sci 2011; 124:3753-3759.

73 Tan W, Palmby TR, Gavard J, Amornphimoltham P, Zheng Y, Gutkind JS. An essential role for Rac1 in endothelial cell function and vascular development. FASEB J 2008; 22:1829-1838.

74 Huotari J, Helenius A. Endosome maturation. EMBO J 2011; 30:3481-3500.

75 Hama H, Tall GG, Horazdovsky BF. Vps9p is a guanine nucleotide exchange factor involved in vesicle-mediated vacuolar protein transport. J Biol Chem 1999; 274:15284-15291.

76 Gerez L, Mohrmann K, van Raak M, et al. Accumulation of rab4GTP in the cytoplasm and association with the peptidylprolyl isomerase pin1 during mitosis. Mol Biol Cell 2000; 11:2201-2211.

77 Rottner K, Hall A, Small JV. Interplay between Rac and Rho in the control of substrate contact dynamics. Curr Biol 1999; 9:640-648.

78 Sorkin A, von Zastrow M. Endocytosis and signalling: in- 
tertwining molecular networks. Nat Rev Mol Cell Biol 2009; 10:609-622.

79 Rantala JK, Pouwels J, Pellinen T, et al. SHARPIN is an endogenous inhibitor of $\beta 1$-integrin activation. Nat Cell Biol 2011; 13:1315-1324.

80 Guidolin D, Vacca A, Nussdorfer GG, Ribatti D. A new image analysis method based on topological and fractal parameters to evaluate the angiostatic activity of docetaxel by using the Matrigel assay in vitro. Microvasc Res 2004; 67:117-124.

81 Roberts M, Barry S, Woods A, van der Sluijs P, Norman J. PDGF-regulated rab4-dependent recycling of alphavbeta3 integrin from early endosomes is necessary for cell adhesion and spreading. Curr Biol 2001; 11:1392-1402.

(Supplementary information is linked to the online version of the paper on the Cell Research website.)

(a) This work is licensed under the Creative Commons Attribution-NonCommercial-No Derivative Works 3.0 Unported License. To view a copy of this license, visit http:// creativecommons.org/licenses/by-nc-nd/3.0 